\title{
Dual-tasking with simple linguistic tasks: Evidence for serial processing
}

\author{
Amie Fairs $^{\mathrm{a}, \mathrm{b}, *}$, Sara Bögels ${ }^{\mathrm{a}, \mathrm{c}}$, Antje S. Meyer ${ }^{\mathrm{a}, \mathrm{c}}$ \\ ${ }^{a}$ Max Planck Institute for Psycholinguistics, P.O. Box 310, 6500AH Nijmegen, the Netherlands \\ ${ }^{\mathrm{b}}$ International Max Planck Research School for Language Sciences, P.O. Box 310, 6500AH Nijmegen, the Netherlands \\ ${ }^{\mathrm{c}}$ Donders Institute for Brain, Cognition and Behaviour, P.O. Box 9010, 6500GL Nijmegen, the Netherlands
}

\section{A R T I C L E I N F O}

\section{Keywords:}

Dual-task interference

Semantic interference

Picture naming

Psychological refractory period

Task choice

\begin{abstract}
A B S T R A C T
In contrast to the large amount of dual-task research investigating the coordination of a linguistic and a nonlinguistic task, little research has investigated how two linguistic tasks are coordinated. However, such research would greatly contribute to our understanding of how interlocutors combine speech planning and listening in conversation. In three dual-task experiments we studied how participants coordinated the processing of an auditory stimulus (S1), which was either a syllable or a tone, with selecting a name for a picture (S2). Two SOAs, of $0 \mathrm{~ms}$ and $1000 \mathrm{~ms}$, were used. To vary the time required for lexical selection and to determine when lexical selection took place, the pictures were presented with categorically related or unrelated distractor words. In Experiment 1 participants responded overtly to both stimuli. In Experiments 2 and 3, S1 was not responded to overtly, but determined how to respond to S2, by naming the picture or reading the distractor aloud. Experiment 1 yielded additive effects of SOA and distractor type on the picture naming latencies. The presence of semantic interference at both SOAs indicated that lexical selection occurred after response selection for S1. With respect to the coordination of S1 and S2 processing, Experiments 2 and 3 yielded inconclusive results. In all experiments, syllables interfered more with picture naming than tones. This is likely because the syllables activated phonological representations also implicated in picture naming. The theoretical and methodological implications of the findings are discussed.
\end{abstract}

\section{Introduction}

A key issue in cognitive psychology is how different cognitive processes are coordinated with one another. This issue has often been investigated in dual-task paradigms, where on each trial participants are asked to respond to two stimuli presented in quick succession. Many dual-task studies have investigated combinations of a linguistic and a non-linguistic task (e.g., Ayora et al., 2011; Cleland, Tamminen, Quinlan, \& Gaskell, 2012; Cook \& Meyer, 2008; Ferreira \& Pashler, 2002). There is much less research concerning combinations of two linguistic tasks. Such research is, however, of great importance for psycholinguistics. This is because language is most often used in conversation, where upcoming speakers can begin to plan their utterances while they are still listening to their interlocutor (Barthel, Sauppe, Levinson, \& Meyer, 2016; Bögels, Magyari, \& Levinson, 2015; Levinson \& Torreira, 2015; Sjerps \& Meyer, 2015). While such linguistic dualtasking is often seen as essential for holding a conversation, the underlying skills are still poorly understood. For instance, it is currently unknown how utterance comprehension is affected by concurrent speech planning, or how speech planning is affected by concurrent comprehension. Evidence concerning these important issues can come from dual-task studies with two linguistic tasks. In the present study, we used dual-task paradigms to examine how the processing of a syllable or a tone was combined with picture naming. This research had two goals: (1) to explore the usefulness of dual-task paradigms for research on the coordination of speaking and listening; specifically to determine whether previous key findings of studies using non-linguistic stimuli could be replicated with linguistic stimuli, and (2) to contribute to psycholinguistic theories of conversation; specifically to examine how a key component of speech planning, lexical selection, could be combined with the processing of a spoken syllable.

We used two paradigms, the psychological refractory period (PRP) paradigm (Pashler, 1994), and the task choice (TC) paradigm (Besner \& Care, 2003). Both paradigms used the same stimuli, namely one of two tones or syllables (stimulus $1, \mathrm{S1}$ ) and a picture with a written distractor word (stimulus 2, S2), but they differed in the tasks. In the PRP experiment (Experiment 1) two overt responses were required: identification of the tone or syllable and naming of the picture. In the TC experiments (Experiments 2 and 3) no overt response was required for S1. Instead, S1 instructed the participant in how to respond to S2, by

\footnotetext{
* Corresponding author at: Max Planck Institute for Psycholinguistics, P.O. Box 310, 6500AH Nijmegen, the Netherlands.

E-mail address: amie.fairs@mpi.nl (A. Fairs).
} 


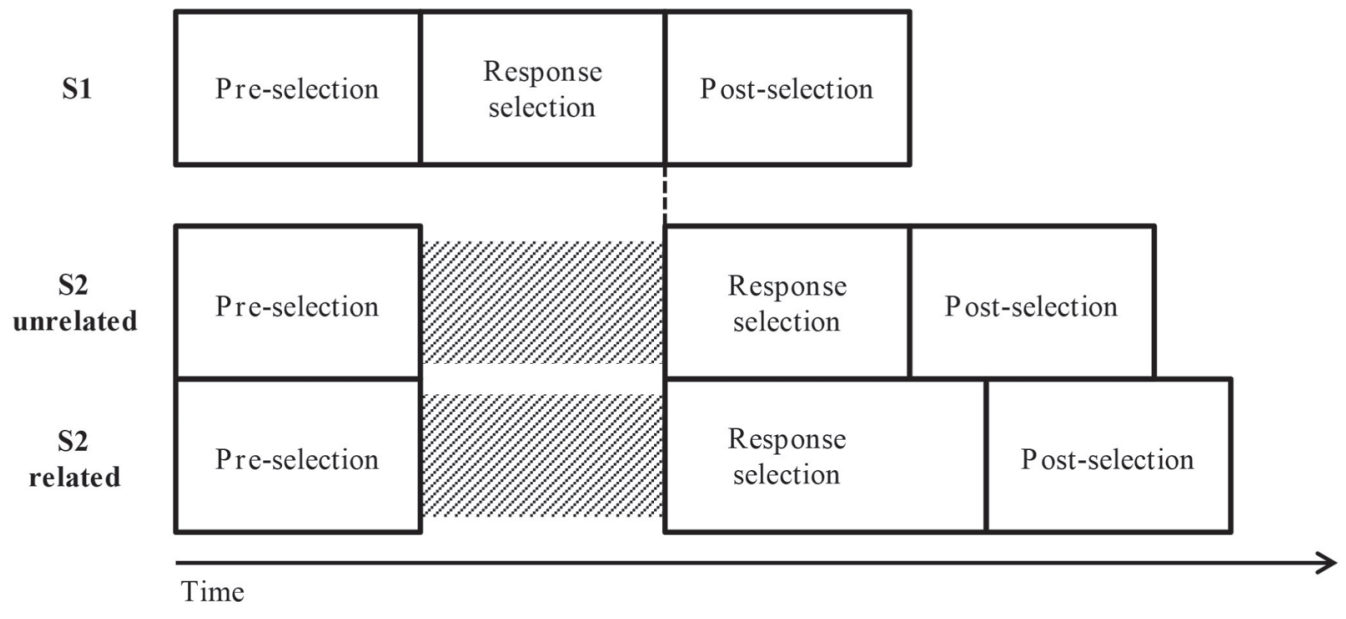

Fig. 1. Diagram of tone/syllable identification ( $\mathrm{S} 1$; task 1 - top bar) and picture naming with distractors (S2; task 2 - bottom two bars) at SOA $0 \mathrm{~ms}$. The pre-selection stages of the two tasks are carried out simultaneously. The response selection stage of $\mathrm{S} 1$ is carried out before the response selection stage of S2. Semantic interference occurs in the response selection stage, with a longer stage for related compared to unrelated stimuli. The greyed area shows the cognitive slack. naming the picture or by reading aloud the distractor. Earlier PRP experiments (Piai, Roelofs, \& Schriefers, 2014; Schnur \& Martin, 2012) using non-linguistic S1 found that participants strongly preferred to postpone lexical selection until after response selection of S1. In contrast, earlier TC experiments have shown that the initial processing of non-linguistic S1 can occur in parallel with lexical selection. Our aim was to determine whether we would replicate these patterns with both non-linguistic and linguistic S1. One hypothesis is that syllables and tones should be processed in the same way. An alternative is that syllables, being linguistic stimuli, may automatically activate associated linguistic representations and consequently interfere more with lexical selection than tones, and/or that the processing of a linguistic S1 may be hampered more by concurrent picture naming than processing of a non-linguistic S1. Because of such cross-talk participants may adopt more sequential processing strategies when syllables rather than tones are used as S1. In the remainder of this Introduction we focus on the predictions for the PRP experiment (Experiment 1). The predictions for the TC paradigm are laid out later (Experiments 2 and 3).

Experiment 1 was a near-replication and extension of Experiment 4 conducted by Piai et al. (2014), which we describe in some detail. On each trial of Piai et al.'s study, participants carried out a response to a tone (S1) and named a picture (S2). The stimulus onset asynchrony (SOA) between tone and picture onset was $0 \mathrm{~ms}$ or $1000 \mathrm{~ms}$. Piai et al. (2014) manipulated the difficulty of lexical selection by combining the pictures with written distractor words that were categorically related to the picture names (as in "deer-rabbit") or unrelated. Numerous studies have demonstrated that categorically related distractors slow down picture naming compared to unrelated ones (e.g., Damian \& Martin, 1999; Roelofs, 2003; Schriefers, Meyer, \& Levelt, 1990). The difference in naming latencies between the related and unrelated distractor conditions is termed the semantic interference effect (Glaser \& Düngelhoff, 1984; Schriefers et al., 1990) and is attributed to competition between distractor and picture names arising during lexical selection (Roelofs, 1992, 2003). Briefly, when a picture-word compound is seen, the written distractor and picture activate their associated lexical representations in parallel. Due to mutual activation between categorically related lexical representations, lexical selection for the target name is hampered more by a related compared to an unrelated distractor, as it takes longer to resolve competition.

The main question addressed by Piai et al. (2014) was when lexical selection occurred relative to the selection of the response to the tone. Relevant evidence came from comparing the interference effects at the two SOAs. At the $1000 \mathrm{~ms}$ SOA, response selection for the tone and lexical selection were most likely carried out in sequence. Consequently, the usual semantic interference effect should be observed. In contrast, when the tone and picture were presented simultaneously, response selection for the tone and lexical selection could be coordinated in different ways. Dual-task theories often distinguish three task stages: pre-selection, response selection, and post-selection (Meyer \& Kieras, 1997; Pashler, 1994; Pashler \& Sutherland, 1998; Tombu \& Jolicœur, 2003). Response selection constitutes a processing bottleneck; that is, only one response can be selected at a time (Pashler, 1984, 1994). This bottleneck has been assumed to be structural (Pashler, 1994) or strategic (Meyer \& Kieras, 1997). ${ }^{1}$ In contrast to response selection, the pre-selection and post-selection processes for two tasks can run in parallel with any other stage.

To return to Piai et al.'s study, if lexical selection is part of preselection processes, it should occur in parallel with pre-selection and response selection processes for the tone. Any competition between target and distractor should be resolved during the "cognitive slack" (Pashler, 1994), i.e. the time that lexical selection waits until the response to the tone has been selected. Therefore at the $0 \mathrm{~ms}$ SOA, the semantic interference effect should be absent or much reduced compared to the effect seen at the $1000 \mathrm{~ms}$ SOA. In contrast, if lexical selection is part of response selection or post-selection processes (see Fig. 1), there is no cognitive slack to absorb the semantic interference effect. Consequently, the effect should be as strong at the $0 \mathrm{~ms}$ as at the $1000 \mathrm{~ms}$ SOA. This is because in both cases, lexical selection occurs after response selection for the tone.

Piai et al. (2014)'s results supported the latter hypothesis. Participants were overall slower to name the pictures at the $0 \mathrm{~ms}$ than at the $1000 \mathrm{~ms} \mathrm{SOA}$, and slower in the related than in the unrelated distractor condition, and these effects were additive. In other words, the interference effect was not absorbed into cognitive slack at the $0 \mathrm{~ms}$ SOA. This pattern of results is consistent with the pattern seen in a number of other studies using the same paradigm (Ayora et al., 2011; Ferreira \& Pashler, 2002; Piai, Roelofs, \& Schriefers, 2011; Piai et al., 2014; Schnur \& Martin, 2012; but see Dell'Acqua, Job, Peressotti, \& Pascali, 2007). It supports the view that the semantic interference effect does not arise prior to, but during or after response selection. It also implies that participants strongly preferred to select the responses to the tone and the picture in sequence.

The main question for Experiment 1 of the present study was whether we would observe the same pattern of results as Piai et al. (2014) when we combined picture naming with tone identification, as they had done, and with syllable identification. Thus, in addition to SOA ( $0 \mathrm{~ms}$ and $1000 \mathrm{~ms}$ ) and relatedness between target and distractor, we varied whether S1 was one of two syllables or one of two tones (S1 type). An obvious hypothesis is that the nature of S1 should not affect

\footnotetext{
${ }^{1}$ Other theories assume no such bottleneck. Response selection of two tasks can be carried out in parallel, but posit a finite amount of capacity which is shared between tasks (Tombu \& Jolicœur, 2003).
} 
the pattern of interference. At the $1000 \mathrm{~ms}$ SOA the response to S1 should be selected well before S2 appears, regardless of the type of S1. At the $0 \mathrm{~ms}$ SOA the pre-selection processes for S1 and S2 should run in parallel but the responses should be selected sequentially, again regardless of the type of S1.

Alternatively, one might expect to find effects of S1 type. This is because the syllables should activate matching and similar phonological representations (Luce \& Pisoni, 1998; Marslen-Wilson, 1987) and possibly word meanings (Gaskell \& Marslen-Wilson, 2002). If, as recently has been proposed (Miozzo, Pulvermüller, \& Hauk, 2015; Strijkers, Costa, \& Pulvermüller, 2017), pictures rapidly activate phonological information, interference may arise affecting the processing of the syllables, which may slow down syllable compared to tone processing. The same holds if written distractor words rapidly activate associated phonological forms (Brown, Joneleit, Robinson, \& Brown, 2002). In other words, when speakers prepare to name pictures, concurrently presented syllables might be harder to identify than tones. In addition, there may be effects of S1 type on picture naming. Syllables should not interfere more than tones with the visual and conceptual processing of the pictures, but additional interference may arise during linguistic encoding. In particular, interference in phonological encoding of the picture names may arise (Schriefers et al., 1990). This would lead to longer picture naming latencies when syllables compared to tones are used as S1.

The prediction that syllables should interfere more with picture naming than tones is consistent with results of a recent PRP study by Fargier and Laganaro (2016), where S1 were tones or syllables and S2 pictures. $^{2}$ As task 1 participants pressed a button to respond to one of five tones or syllables (a go/no-go task; only no-go trials were analysed), and as task 2 they named the picture. Fargier and Laganaro (2016) found longer picture naming latencies in the syllable than the tone condition. ERP modulations in the syllable condition around $350 \mathrm{~ms}$ before speech onset suggested that this effect arose during the phonological encoding of the picture names. The interpretation of these results is complicated by the fact that the syllables were existing words of the participants' native language, that only a single SOA $(300 \mathrm{~ms})$ was used, and that the manual response latencies were not recorded. Nevertheless, the results point towards specific linguistic interference arising from syllable compared to tone processing on picture naming.

\section{Experiment 1: PRP paradigm}

Experiment 1 tested dual-tasking in a PRP paradigm. Participants carried out syllable or tone identification as task 1, with a button press response, and named pictures with distractors as task 2 . Two SOAs were tested $(0 \mathrm{~ms}$ and $1000 \mathrm{~ms})$. The distractor words were categorically related or unrelated to the picture names. In this experiment we had two aims: 1) to replicate the finding of semantic interference at both SOAs with S1 as tones (e.g., Piai et al., 2014; Schnur \& Martin, 2012); and 2) to investigate whether a similar pattern held with S1 syllables. Participants additionally carried out a single S1 identification task block before the dual-tasking trials to practice tone/syllable identification.

\subsection{Methodology}

\subsubsection{Participants}

36 participants $(\mathrm{M}=23$ years, $\mathrm{SD}=3.5,31$ female) were recruited from the Max Planck Participant Database. All self-reported as righthanded, with no language, sight or hearing disorders. Participants were

\footnotetext{
${ }^{2}$ A study by Paucke et al. (2015) tested two production tasks concurrently, where two pictures were displayed side by side. Task 1 was picture naming of the first picture and task 2 was phoneme detection in the planned name of the second picture.
}

paid $€ 12$ for participation, and were given sweets to motivate them to stay on task. The experiment was granted ethical approval by the Radboud University Social Sciences ethics committee in accordance with the Declaration of Helsinki.

\subsubsection{Materials}

Two sine wave pure tones were generated using Audacity (Audacity team 2012) at $300 \mathrm{~Hz}$ (low tone) and $800 \mathrm{~Hz}$ (high tone). Two Dutch syllables, [a:k] and [i:k], referred to as 'aak' and 'iek', were recorded by a female native Dutch speaker. The syllables were of the form VVC so that they were maximally discriminable from the onset of the syllable, and so they roughly matched the tones in height discrimination ([a:] is 'low' and [i:] is 'high' in the vowel trapezium). Participants were made aware of the high/low mapping for both tones and syllables. All sounds were $460 \mathrm{~ms}$ long. The vowel length of 'aak' was $263 \mathrm{~ms}$ and the vowel length of 'iek' was $264 \mathrm{~ms}$. Both 'aak' and 'iek' were on average $222 \mathrm{~Hz}$ over the entire syllable. Tones and syllables were equalised to $70 \mathrm{~dB}$.

The tones and syllables were pre-tested to ensure equal reaction times (RTs) as a proxy for difficulty. In a pre-test with 16 participants there was no significant difference between RTs to the tones and syllables $\left(\mathrm{M}_{\text {Tone }}=497 \mathrm{~ms}(\mathrm{SD}=216 \mathrm{~ms}), \mathrm{M}_{\text {Syllable }}=511 \mathrm{~ms}(\mathrm{SD}=191 \mathrm{~ms})\right.$, $\mathrm{t}=-0.72$ ). The same stimuli were also used in a previous experiment (Fairs, Bögels, and Meyer, in preparation) where RTs in the single identification task were almost identical between conditions $\left(\mathrm{M}_{\text {Tone }}=443.7 \mathrm{~ms} \quad(\mathrm{SD}=157 \mathrm{~ms}), \mathrm{M}_{\text {Syllable }}=444.4 \mathrm{~ms} \quad(\mathrm{SD}=178 \mathrm{~ms})\right.$, $\mathrm{t}=0.37,33$ participants). We concluded that the syllables and tones were matched in difficulty.

The picture materials were taken from Piai et al. (2014). The pictures were 32 white line drawings on a black background, and each picture was shown once with a related distractor and once with an unrelated distractor. Distractors were part of the response set. These 64 picture-distractor pairs were shown once at each SOA with each tone and each syllable. This led to 512 trials, 256 with each S1 (tone or syllable). A further 20 images with distractor words were selected for practice trials. These images were not used in the experiment proper. Of the practice images, only two distractors belonged to the response set (i.e. were also practice picture names). All images were sized $300 \times 300$ pixels and centered in the middle of the screen. The distractor words were printed in white size 36 arial font in the center of the picture.

\subsubsection{Design}

Participants carried out single tone/syllable identification for 60 trials before moving on to the dual-task trials. Each participant had a unique input file with a pseudo-random order for the single task, generated with the Mix programme (van Casteren \& Davis, 2006), with the constraint that the same tone/syllable could repeat at most five times.

For the dual-task trials, task 1 was tone/syllable identification. Button press latencies were measured as the task 1 response. Task 2 was picture naming (with a written distractor). Picture naming latencies from picture onset were measured as the task 2 response. Stimulus 1 type (S1 type; tone or syllable) was a within-participant factor, and blocked, and this block order was counterbalanced between participants. Two SOAs were tested: $0 \mathrm{~ms}$ and $1000 \mathrm{~ms}$. SOA and relatedness were within-participant factors, and were pseudo-randomly presented within each block. Each participant had a unique input file with the following constraints: a) maximum of three repetitions of SOA; b) maximum of three repetitions of relatedness; c) minimum distance of 20 pictures between each picture repetition; d) maximum of five repetitions of the same $\mathrm{S} 1$; e) minimum distance of two written distractors between each distractor repetition.

256 experimental trials were shown with each S1 type. An additional six warm-up trials (from the practice set) were added: two at the beginning of each block, and two after each break, which were removed prior to analysis. Participants practiced the dual-task on a separate set of 24 practice trials, which (aside from the six warm-up trials) were not 
displayed during experimental trials.

\subsubsection{Apparatus}

The experiment was presented on a Benq monitor using the software Presentation (version 16.5, www.neurobs.com). Participants listened to the tones/syllables through Sennheiser HD437 headphones. A custom made quiet button box (created at the MPI, using small microphones rather than buttons) recorded button presses to task 1. A Sennheiser microphone recorded participants' speech to task 2 and the vocal response on each trial was recorded in an individual sound file of $3000 \mathrm{~ms}$ by the Presentation software.

\subsubsection{Procedure}

Participants were first familiarised with each practice and experimental picture in the experiment. All pictures were presented in a random order. Each picture was displayed slightly above the center of the screen with the name of the picture written underneath. Participants were instructed to look at the picture before reading the name out loud and to remember the picture name. Once the participant had read the name aloud, the experimenter displayed the next picture.

Participants were then familiarised with each tone/syllable. After this, participants carried out 60 single task trials. Each trial began with a fixation cross for $700 \mathrm{~ms}$ followed immediately by the auditory stimulus. Participants pressed the left button for the low tone/aak syllable, and the right button for the high tone/iek syllable. The trial ended when a button was pressed or after $1500 \mathrm{~ms}$ if there was no response. A blank screen was then presented for $500 \mathrm{~ms}$ before the onset of the next trial. The response buttons were not counterbalanced across participants to avoid disrupting any inherent stimulus-response mappings (i.e. low is left, high is right).

Participants then practiced the dual-task. For the first six trials of the practice block, participants saw only pictures with distractors, and were instructed to name the pictures. After this, each dual-task trial began with a fixation cross for $700 \mathrm{~ms}$. In trials with SOA $0 \mathrm{~ms}$, the auditory stimulus and visual stimulus (S1 snd S2) were displayed at the same time. The S2 stimulus remained on screen for $500 \mathrm{~ms}$ before being replaced with a blank screen for $1750 \mathrm{~ms}$. In trials with SOA $1000 \mathrm{~ms}$, the S1 stimulus was presented first and $1000 \mathrm{~ms}$ after auditory onset the S2 stimulus was displayed for $500 \mathrm{~ms}$, with a blank screen displayed afterwards for $1750 \mathrm{~ms}$. Experimental trials were presented with exactly the same structure. Participants were instructed to respond to the auditory stimulus with a button press before naming the picture.

Participants took a break between each new task of the experiment, and were given two breaks (one after 90 trials and the other after 180 trials) during the dual-task trials. In the middle of the experiment, participants were encouraged to have a longer break and to leave the testing booth. The experimenter controlled when the participant would start the next block of the experiment. After the experiment, participants were fully debriefed. The entire testing session took approximately $75 \mathrm{~min}$.

\subsection{Results}

\subsubsection{Pre-processing and analysis}

Data were checked for errors. A trial contained an error if the wrong button was pressed for the tone/syllable, if there were any hesitations or disfluencies in the speech recording, if the picture name was incorrect, or if participants named the picture before pressing the button. Any participant who made $>20 \%$ errors in either the single or the dual-task was removed. 4 participants were removed from the dataset for having extremely long RTs in the single task $(\mathrm{N}=1)$, not following task instructions $(\mathrm{N}=1)$, or having $>20 \%$ errors in picture naming trials $(\mathrm{N}=2)$. This left analysable datasets from 32 participants.

The first 30 trials of the single task were removed as practice trials before error checking. Incorrect responses and RTs shorter than $200 \mathrm{~ms}$ were removed $(\mathrm{N}=45,2.3 \%)$. For the dual-task, button press $\mathrm{RTs}$ were automatically measured by the experimental software. Speech latencies were manually measured using Praat (Boersma et al., 2002) for each trial. Before error checking, warm-up trials and any trials with an RT of $<200 \mathrm{~ms}$ were removed. $8.2 \%(\mathrm{~N}=1386)$ of all dual-task trials were removed as errors.

Data were analysed with linear mixed effects models (lme4 package; Bates, Maechler, Bolker, Walker, et al., 2014) using R (R Core Team, 2014). The maximal random structure that would lead to convergence (after adjusting simulation runs and the optimizer) is presented (Barr, Levy, Scheepers, \& Tily, 2013). All RTs were log-transformed (base 10) prior to analysis to reduce skew, continuous variables were centered and categorical variables were sum-to-zero contrast coded. Error data were analysed with a binomial logit mixed model, and with the control variable trial centered and scaled. The same models were also run on the raw RT data to confirm that effects were not masked by the logtransformation. Results from these models are presented in the Supplementary Materials (see Appendix). All raw RT models found the same pattern of results. We took $|t|>2$ to be our marker of significance. 95\% confidence intervals are reported, calculated using the 'profile' option in the confint.merMod function in lme4. All post-hoc tests were carried out with the lsmeans package (Lenth, 2016).

\subsubsection{Experiment 1: Single identification task}

For the single identification task, mean RTs to the tones were $410 \mathrm{~ms}(\mathrm{SD}=125 \mathrm{~ms}$ ), and mean RTs to the syllables were $450 \mathrm{~ms}$ $(\mathrm{SD}=137 \mathrm{~ms})$. The difference between conditions was not predicted as explained in Materials (Section 2.1.2).

A linear mixed effect model with log RT as the dependent variable, S1 type as an experimental fixed effect, and fixed control effects of block order (was the tone or syllable block carried out first), trial, and a block order by S1 type interaction was modelled. The random effects structure included a random intercept by participant and random slope of $\mathrm{S} 1$ type by participant.

For the fixed control effects, we found an effect of trial (estimate $=-0.0006, \mathrm{SE}=0.0002, t=-3.2$, CI $[-0.001-0.0003]$ ) as participants sped up through the block. The main effect of block order was not significant $(t<1)$ but the block order by S1 type interaction was significant (estimate $=0.017, \mathrm{SE}=0.007, t=2.58$, CI $[0.004$ 0.03]). This was driven by the fact that participants who carried out the syllable identification block before the tone identification block had a $78 \mathrm{~ms}$ difference between single task RTs $\left(\mathrm{M}_{\text {syllable }}=462 \mathrm{~ms}(\mathrm{SD}=\right.$ $125 \mathrm{~ms}), \mathrm{M}_{\text {tone }}=384 \mathrm{~ms}$ ( $\left.\mathrm{SD}=108 \mathrm{~ms}\right)$ ), whereas participants with the reverse order had only a $7 \mathrm{~ms}$ difference between RTs $\left(\mathrm{M}_{\text {tone }}=433 \mathrm{~ms}\right.$ $\left.(\mathrm{SD}=155 \mathrm{~ms}), \mathrm{M}_{\text {syllable }}=440 \mathrm{~ms}(\mathrm{SD}=135 \mathrm{~ms})\right)$. We also found a main effect of S1 type (estimate $=0.023$, SE $=0.007, t=3.44$, CI $[0.01$ $0.036]$ ), indicating that responses to syllables were slower than to tones, even when controlling for the block order effect.

A linear mixed effects model of the error data $(2.3 \%$ of the data; $1.7 \%$ in syllable identification and $3.1 \%$ in tone identification) was conducted with the same model structure as for the RT data. We found no significant effects (all z's $<2$, all $p$ 's $>0.1$ ).

\subsubsection{Experiment 1: Task 1 RTs - tone/syllable identification}

Fig. 2 shows the mean RTs for task 1 by S1 type and SOA in the dualtask. At SOA $0 \mathrm{~ms}$, syllables were responded to $54 \mathrm{~ms}$ slower than tones. At SOA $1000 \mathrm{~ms}$, syllables were responded to $38 \mathrm{~ms}$ slower than tones.

A linear mixed effects model run on the task 1 data included log transformed RT as the dependent variable. Fixed control predictors included trial, block order, and a block order by S1 type interaction. The fixed experimental predictors were SOA, S1 type, relatedness (related or unrelated distractor word), and a S1 type by SOA interaction. The random effects structure included random intercepts by participant and item, and random slopes of SOA, S1 type and relatedness by participant.

We found no effect of block order or significant block order by S1 type interaction (both t's $<2$ ). There was a significant effect of trial 


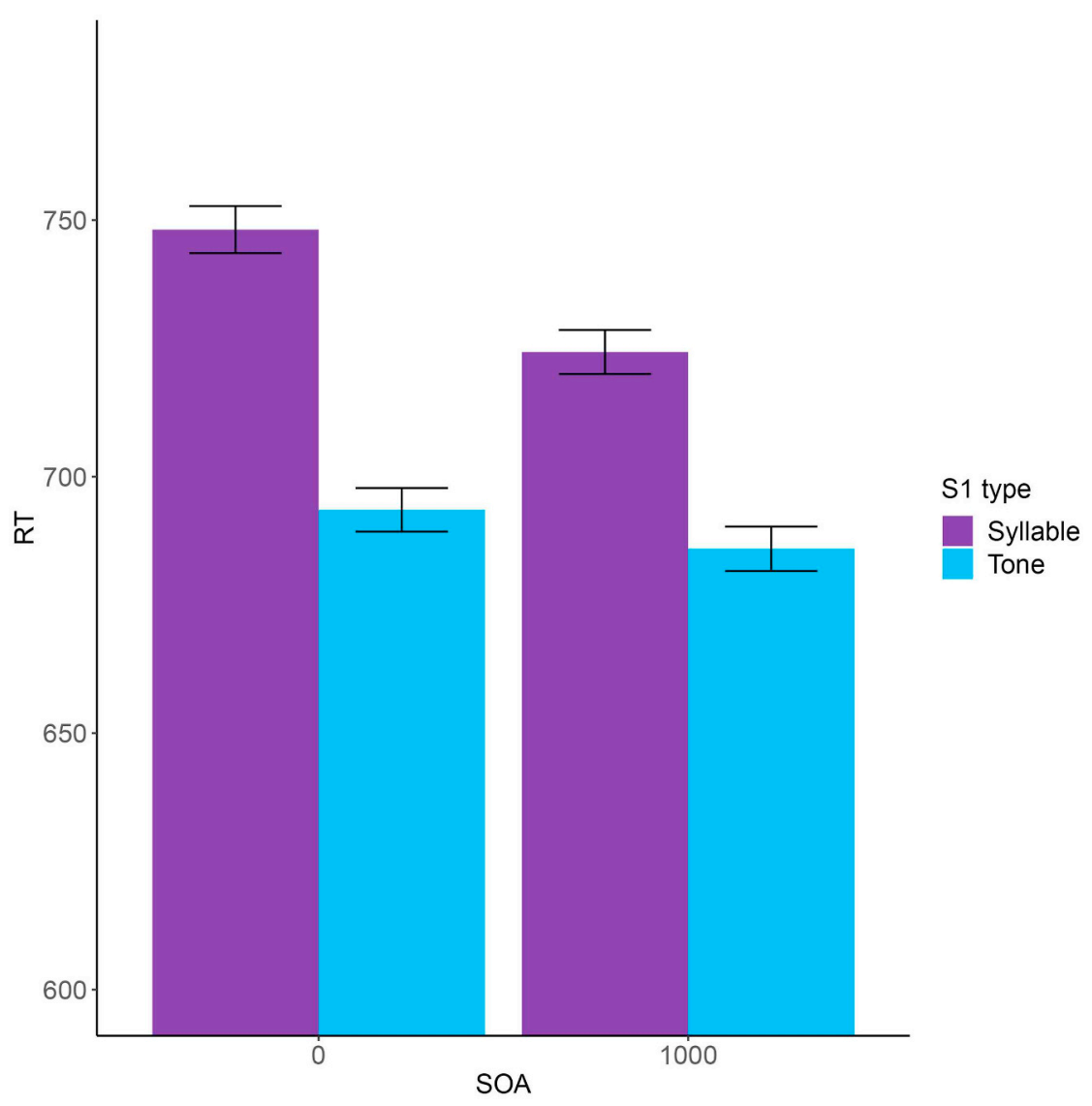

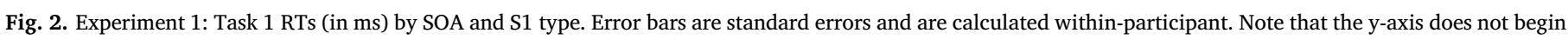
at zero.

such that participants sped up within each block (estimate $=-1.52 \mathrm{e}$ $04, \mathrm{SE}=1.31 \mathrm{e}-05, t=-11.62$, CI $[-0.0002-0.0001])$. There was no main effect of relatedness (estimate $=2.5 \mathrm{e}-04, \quad \mathrm{SE}=1.03 \mathrm{e}-03$, $t=0.24$, CI [ $-0.0020 .002])$. There was a significant effect of S1 type (estimate $=1.49 \mathrm{e}-02, \mathrm{SE}=4.42 \mathrm{e}-03, t=3.36$, CI [0.006 0.024]), such that participants responded more slowly to syllables than tones. There was no effect of SOA (estimate $=7.09 \mathrm{e}-03, \mathrm{SE}=4.81 \mathrm{e}-03, t=1.47$, CI [ $-0.0020 .017]$ ), meaning that participants responded equally fast at both $0 \mathrm{~ms}$ and $1000 \mathrm{~ms}$, and no significant S1 type by SOA interaction (estimate $=1.78 \mathrm{e}-03, \mathrm{SE}=9.87 \mathrm{e}-04, t=1.8$, CI $[-0.00020 .004]$ ), indicating that the RT difference between syllables and tones was of the same magnitude at both SOAs.

$2.6 \%$ of the data were task 1 errors. The error counts are presented in Table 1. A linear mixed effects model of this error data was conducted with the same model structure as for the RT data except with no S1 type random slope. There was an effect of trial (estimate $=0.08$, $\mathrm{SE}=0.04, \mathrm{z}=2.22, p=.03$ ), as participants made more errors as the experiment went on. There was an effect of relatedness (estimate $=-0.19, \mathrm{SE}=0.05, \mathrm{z}=-3.77, p<.001$ ), as participants made more errors in the related condition than the unrelated condition. There were also significant interactions between S1 type and block

Table 1

Experiment 1: Error proportions in task 1 RTs by S1 type, SOA and relatedness.

\begin{tabular}{llllll}
\hline & \multicolumn{2}{l}{ S1 syllable } & & & \multicolumn{2}{l}{ S1 tone } \\
\cline { 2 - 3 } \cline { 5 - 6 } \cline { 5 - 6 } & SOA 0 ms & SOA 1000 ms & & SOA 0 ms & \multirow{2}{*}{ SOA 1000 ms } \\
\hline Related & 0.06 & 0.05 & & 0.05 & 0.06 \\
Unrelated & 0.04 & 0.04 & & 0.04 & 0.05 \\
\hline
\end{tabular}

order (estimate $=-0.12, \mathrm{SE}=0.06, \mathrm{z}=-2.2, p=.03$ ) and S1 type and SOA (estimate $=-0.1, \mathrm{SE}=0.04, \mathrm{z}=-2.53, p=.01$ ). The first interaction arose because there was no difference in error proportions in the order syllable-tone $(\mathrm{z}=0.8, p=.42)$, but more errors in syllable identification than tone identificationin the order tone-syllable $(\mathrm{z}=-2.08, p=.04)$. There was a higher proportion of errors at SOA $0 \mathrm{~ms}$ in the syllable compared to the tone condition $(\mathrm{z}=-2.16$, $p=.03$ ), whereas the proportion of errors by S1 type at SOA $1000 \mathrm{~ms}$ was similar $(\mathrm{z}=0.53, p=.6)$. These results parallel those found in the RT analysis. There was no evidence for a speed-accuracy trade off as higher numbers of errors were made in the conditions which have the slowest RTs.

\subsubsection{Experiment 1: Task 2 latencies - picture naming}

Fig. 3 shows the mean latencies for task 2 by S1 type, SOA and relatedness in the dual-task. The descriptive relatedness effect at SOA $0 \mathrm{~ms}$ with S1 syllables was $36 \mathrm{~ms}$ and with $\mathrm{S} 1$ tones was $29 \mathrm{~ms}$. At SOA $1000 \mathrm{~ms}$ the effect with S1 syllables was $19 \mathrm{~ms}$ and with $\mathrm{S} 1$ tones was $22 \mathrm{~ms}$.

A linear mixed effects model included log-transformed task 2 latencies as the dependent variable. Fixed control predictors included trial, block order, log-transformed task 1 RTs and a block order by S1 type interaction. Log-transformed task 1 RTs were included as a control predictor. Because participants were explicitly instructed to respond to task 2 after task 1 , and we measure longer RTs to task 1 syllable identification at SOA $0 \mathrm{~ms}$ than tone identification, we would expect that task 2 naming latencies would also be longer at SOA $0 \mathrm{~ms}$ with S1 syllables than S1 tones. We thus included task 1 RTs as a control predictor. The fixed experimental predictors were SOA, S1 type, relatedness, and all interactions. The random effects structure included random intercepts by participant and item, random slopes of SOA, S1 


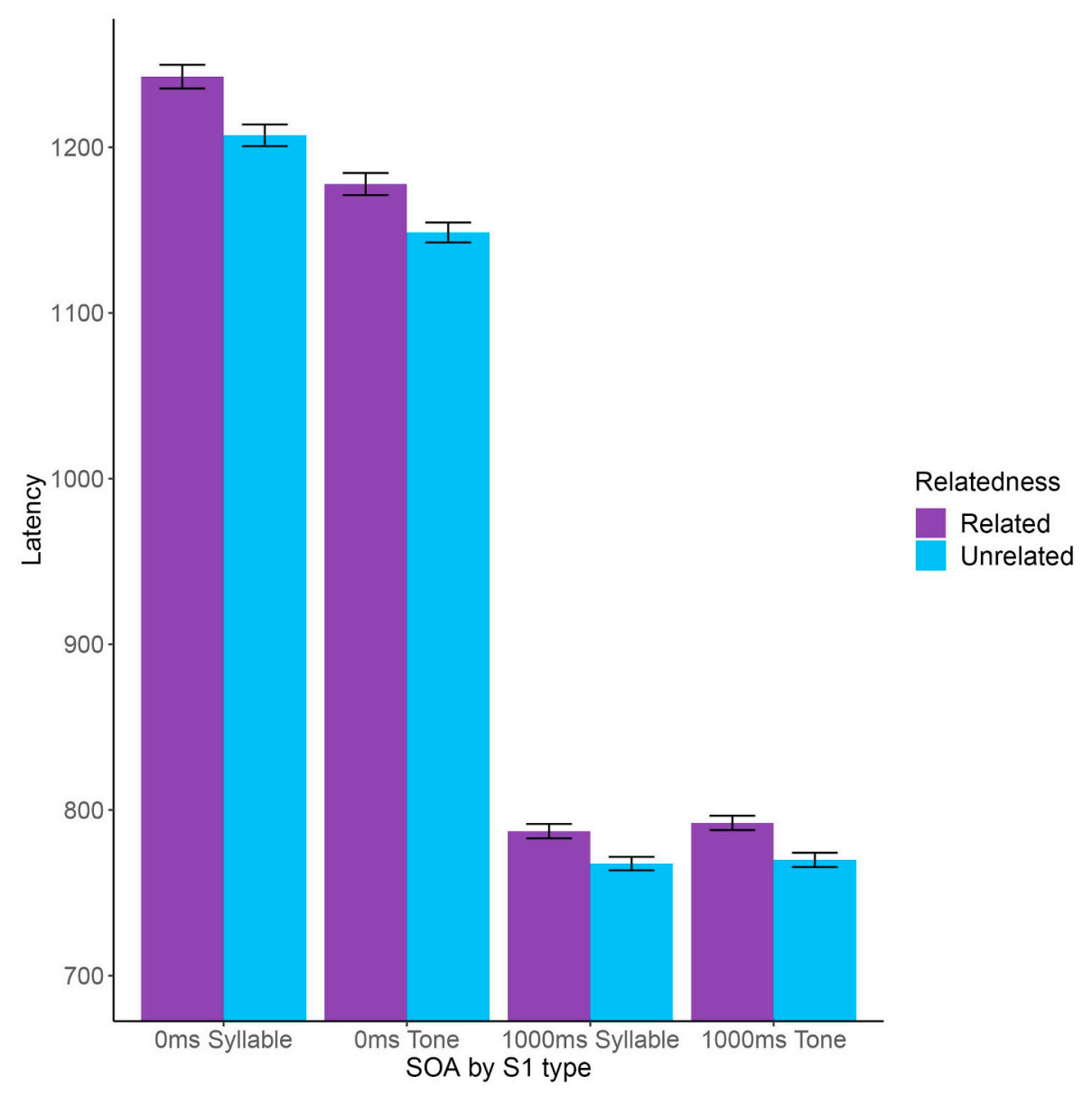

Fig. 3. Experiment 1: Task 2 mean latencies (in ms) by SOA, S1 type and relatedness. Error bars are standard errors and are calculated within-participant. Note that the $y$-axis does not begin at zero.

type and relatedness by participant, and random slopes of SOA and S1 type by item.

All control predictors were significant: trial (estimate $=-6.41 \mathrm{e}-05$, $\mathrm{SE}=7.71 \mathrm{e}-06, t=-8.32$, CI [ $-7.9 \mathrm{e}-05-4.9 \mathrm{e}-05])$, log-transformed task 1 RTs (estimate $=0.28, \mathrm{SE}=4.73 \mathrm{e}-03, t=58.57$, CI [0.27 0.29]), block order (estimate $=1 \mathrm{e}-02, \mathrm{SE}=4.46 \mathrm{e}-03, t=2.25$, CI $[0.001$ $0.02]$ ), and block order by $S 1$ type interaction (estimate $=3.32 \mathrm{e}-03$, $\mathrm{SE}=1.43 \mathrm{e}-03, t=2.33$, CI [4.6e-04 6.2e-03]). As with the single task RT analysis, participants who began the experiment with the tone block had similar naming latencies regardless of S1, whereas participants who began with the syllable block were slower to name pictures with S1 as syllables compared to tones. As the order of blocks was a betweenparticipant variable and due to counter-balancing, this difference was likely due to variation in participants. Importantly, including this predictor as a control means that our experimental variables of interest can be interpreted over and above any counterbalancing effects.

The experimental predictor S1 type was not significant $(t<1)$, meaning that naming latencies were similar regardless of S1 type. The predictors SOA (estimate $=8.79 \mathrm{e}-02, \mathrm{SE}=4.33 \mathrm{e}-03, t=20.29$, CI $[0.0790 .096]$ ) and relatedness (estimate $=5.19 \mathrm{e}-03, \mathrm{SE}=7.98 \mathrm{e}-04$, $t=6.5$, CI $[0.004$ 0.007]) were significant. Participants were significantly slower at SOA $0 \mathrm{~ms}$ than SOA $1000 \mathrm{~ms}$, and participants were significantly slower naming in the related condition compared to the unrelated condition. The interaction between S1 type and SOA was significant (estimate $=5.53 \mathrm{e}-03, \mathrm{SE}=5.78 \mathrm{e}-04, t=9.57$, CI $[0.004$ $0.007]$ ). At $0 \mathrm{~ms}$ latencies with $\mathrm{S} 1$ syllables were slower than with $\mathrm{S} 1$ tones, with a smaller difference at SOA $1000 \mathrm{~ms}$. The SOA by relatedness interaction and relatedness by $\mathrm{S} 1$ type interaction did not reach significance, indicating that the relatedness effect was not different
Table 2

Experiment 1: Error proportions in task 2 latencies by S1 type, SOA and relatedness.

\begin{tabular}{llllll}
\hline & \multicolumn{2}{l}{ S1 syllable } & & & \multicolumn{2}{l}{ S1 tone } & \\
\cline { 2 - 3 } \cline { 5 - 6 } \cline { 5 - 6 } & SOA 0 ms & SOA $1000 \mathrm{~ms}$ & & SOA 0 ms & SOA 1000 ms \\
\hline Related & 0.06 & 0.04 & & 0.05 & 0.06 \\
Unrelated & 0.03 & 0.04 & & 0.03 & 0.04 \\
\hline
\end{tabular}

between SOAs, and the relatedness effect did not differ by S1 type. The three-way interaction also did not reach significance (all t's $<1$ ).

$4.4 \%$ of the data were analysable task 2 errors, presented in Table 2. This excluded unanalysable errors, such as trials with sneezing, yawning, or coughing. A mixed effects model of the analysable error data was conducted with a similar model structure as for the latency data, but without log-transformed task 1 RTs, and with a random slope of relatedness by item. There was a main effect of trial (estimate $=0.14, \mathrm{SE}=0.04, \mathrm{z}=3.62, p<.001$ ) as participants made more errors as the experiment progressed, and a significant interaction between $\mathrm{S} 1$ type and block order (estimate $=-0.13, \mathrm{SE}=0.06$, $\mathrm{z}=-2.39, p=.02$ ). There was also a main effect of relatedness (estimate $=-0.24, \mathrm{SE}=0.06, \mathrm{z}=-3.88, p<.001$ ), as participants made more errors in the related condition than the unrelated condition. The interaction between S1 type and SOA was also significant (estimate $=-0.11, \mathrm{SE}=0.04, \mathrm{z}=-2.71, p=.007)$. With S1 syllables, there was no difference in errors made at SOA $0 \mathrm{~ms}$ compared to SOA $1000 \mathrm{~ms}(\mathrm{z}=-0.55, p=.58)$. With $\mathrm{S} 1$ tones, more errors were made 
at SOA $1000 \mathrm{~ms}$ than SOA $0 \mathrm{~ms}(\mathrm{z}=2.27, p=.02)$. Thus, there is some evidence for a speed-accuracy trade-off, but only with $S 1$ tones.

\subsection{Discussion}

In this experiment we replicated the key findings of the PRP experiment reported by Piai et al. (2014; see also Schnur \& Martin, 2012): We found additive effects of SOA and relatedness on picture naming latencies. In other words, we saw semantic interference effects of equal size at both SOAs. This shows that participants first selected the response to S1 and then selected the name of the picture (see Fig. 1). Importantly, this held for both types of S1, tones and syllables. Thus, the type of S1 did not affect how the participants coordinated the response selection processes for the two stimuli with each other. This is important as it indicates that a key finding of dual-task experiments using tones as S1 was replicated with syllables as S1.

For task 1 (syllable or tone identification), we observed longer RTs to syllables than to tones. This may be due to the syllables being harder to identify than the tones, or, as suggested in the Introduction, due to interference from concurrent picture processing. While we cannot rule out the latter explanation, the former seems more plausible for two reasons. Firstly, in the single identification task, RTs were longer for syllables than tones. This effect was unexpected as a pre-test and previous use of the stimuli had shown no RT difference between these tones and syllables. Secondly, in the analysis of task 1 RTs, the interaction between S1 type and SOA was not significant. Yet, an interference effect from picture naming onto identification should only be observed if the two tasks were carried out in parallel (at the $0 \mathrm{~ms} \mathrm{SOA}$ ), and not if they were performed in sequence (at the $1000 \mathrm{~ms} \mathrm{SOA}$ ). Thus, it appears that the participants found the syllables harder to identify than the tones.

For task 2 (picture naming), we found an interaction of S1 type and SOA. Naming latencies were longer in the syllable condition than in the tone condition at the $0 \mathrm{~ms} \mathrm{SOA}$, but not at the $1000 \mathrm{~ms}$ SOA. Note that in the analysis of picture naming latencies the effect of identification RT was controlled for. Thus, the interaction was not a direct consequence of the longer identification RTs for syllables discussed above. The interaction indicates that concurrent responding to a syllable interfered more with the naming task than concurrent responding to a tone. Thus, we observed cross-talk between similar tasks, as reported in earlier dual-task studies (e.g., Hommel, 1998; Lien, Ruthruff, Hsieh, \& Yu, 2007; Logan \& Schulkind, 2000; Paucke, Oppermann, Koch, \& Jescheniak, 2015). We return to the implications of this finding in the General Discussion.

\section{Experiment 2: Task choice paradigm}

Experiment 1 showed that participants postponed lexical selection until after response selection for task 1 . This is consistent with the view that in dual-task paradigms participants generally prefer to execute response selection processes in sequence rather than in parallel. Theories of dual-task performance commonly assume that the selection stage for a task, where stringent capacity restrictions apply, is preceded by a pre-selection stage, during which multiple cognitive processes can run in parallel (Pashler, 1994; Tombu \& Jolicœur, 2003). A number of studies, using the task choice (TC) paradigm described below, have investigated whether lexical selection for a picture name could occur during the pre-selection stage for a non-linguistic stimulus. In Experiments 2 and 3 we used the same paradigm to study whether lexical selection could occur during the early processing of a tone and a syllable.

The TC study that is most relevant to the present research was conducted by Piai, Roelofs, and Schriefers (2015; see also Janssen, Schirm, Mahon, \& Caramazza, 2008; Mädebach, Oppermann, Hantsch, Curda, \& Jescheniak, 2011). In this study, the same pictures but tones with different frequencies were used as in Experiment 1 reported above.
Tones and pictures were presented with SOAs of $0 \mathrm{~ms}$ and $1000 \mathrm{~ms}$ (Experiment 1) or with SOAs of $0 \mathrm{~ms}$ and $350 \mathrm{~ms}$ (Experiment 2). No overt response to the tone was required. Instead the tone instructed the participant to name the picture or read aloud the distractor word. As Piai et al. (2011) pointed out, in this task participants could carry out some preparation for both verbal responses, but had to suspend these processes at some point and make the task decision (to read aloud the word or name the picture). They proposed that a good suspension point would be just before the initiation of word form retrieval. This is because word form retrieval for picture naming and for reading aloud requires processing capacity and cannot be easily combined with the task decision (Piai et al., 2011, 2015). Thus, planning processes for the picture name would be suspended after lexical selection. If processing the tone and making the task decision take more time than preparing the picture name up to lexical selection, the semantic interference effect should be absorbed into the cognitive slack created by slower task decision processes. Consequently, the effect should be absent at the $0 \mathrm{~ms}$ SOA. In contrast, it should be present at the later SOAs, where the task decision precedes lexical selection. This was exactly the pattern Piai et al. (2015) observed: Semantic interference effects were seen at late SOAs but not at the SOA of $0 \mathrm{~ms}$. This indicates that lexical selection was combined with the pre-selection processes for the tones.

In Experiment 2 we used the TC paradigm with the same materials as in Experiment 1. The aim was to establish whether we would replicate the pattern seen in the TC experiment by Piai et al. (2015) when S1 were tones, and whether this pattern would also be observed when S1 were syllables. As before, a plausible hypothesis is that syllables and tones should not differ in their effects on picture naming latencies. If this is the case, we should only observe an interaction of SOA and relatedness on the picture naming latencies, with the semantic interference effect being absent at the $0 \mathrm{~ms}$ SOA and present at the $1000 \mathrm{~ms}$ SOA. However, Experiment 1 yielded evidence for additional interference of the syllables compared to the tones with the picture naming task, and one might expect this effect to be replicated in the TC paradigm. There should then be a main effect of S1 type, with naming latencies being longer in the syllable than in the tone condition. Finally, because of the additional interference arising in the linguistic condition, participants might choose to schedule the processing of the stimuli differently in the linguistic and non-linguistic condition. While processing may overlap in the tone condition, task choice and lexical selection might occur in sequence in the syllable condition. In the latter case, there would be no slack to absorb the semantic interference effect. Consequently, there should be a three-way interaction, due to additive effects of SOA and relatedness when S1 are syllables, and an interaction of SOA and relatedness when they are tones.

For the word reading task, we did not expect to find a semantic interference effect or an effect of S1 (Glaser \& Düngelhoff, 1984; Piai et al., 2015) since in adults word reading is highly automatized and rather immune to distractor effects. Note that in the TC paradigm there are no task 1 RTs to record.

\subsection{Methodology}

\subsubsection{Participants}

38 participants $(\mathrm{M}=22.6$ years, $\mathrm{SD}=2.4,31$ female $)$ were recruited from the Max Planck Participant Database. All self-reported as right-handed, with no language, sight, or hearing disorders. Participants were paid $€ 12$ for participation, and were given sweets to motivate them to stay on task. The experiment was granted ethical approval by the Radboud University Social Sciences ethics committee in accordance with the Declaration of Helsinki.

\subsubsection{Materials \& apparatus}

The same materials and apparatus were used as in Experiment 1. 


\subsubsection{Design}

The design was the same as Experiment 1, except where indicated. Participants did not carry out any single task identification trials. For the task choice trials, participants were instructed to name the picture if they heard the low tone/'aak' syllable, and read the word if they heard the high tone/'iek' syllable.

One additional constraint was used in generating each participant's input list: there were a minimum of four items between the repetition of any spoken item (either the picture name or the distractor word, depending on what participants should have said on that particular trial).

\subsubsection{Procedure}

The experimental procedure was identical to Experiment 1 except where indicated. Participants began the experiment with the same picture familiarisation phase as in Experiment 1. Participants then practiced the task choice task. Participants first named the picture (with a distractor word present) for four trials, then read the word aloud for two trials, before then being familiarised with the tones/syllables and carrying out 24 practice task choice trials. Written reminders of the tone/syllable mappings were on the table in front of the participants.

\subsection{Results}

\subsubsection{Pre-processing and analysis}

Data were checked for errors in the same way and with the same error criteria as in Experiment 1. Four participants were removed because they did not follow task instructions $(\mathrm{N}=1)$, or had $>20 \%$ errors $(\mathrm{N}=3)$, resulting in analysable datasets from 34 participants. Speech latencies were pre-processed in the same way as Experiment 1.
$6.4 \%(\mathrm{~N}=1119)$ of the data were removed due to errors. Latencies and errors were analysed in the same way as Experiment 1.

\subsubsection{Experiment 2: Naming latencies}

Fig. 4 shows the mean naming latencies by S1 type, SOA, and relatedness. The descriptive relatedness effect at SOA $0 \mathrm{~ms}$ with S1 syllables was $34 \mathrm{~ms}$ and with $\mathrm{S} 1$ tones was $-1 \mathrm{~ms}$. At SOA $1000 \mathrm{~ms}$ the effect with S1 syllables was $27 \mathrm{~ms}$ and with S1 tones was $5 \mathrm{~ms}$.

A linear mixed effects model included log transformed naming latencies as the dependent variable. Fixed control predictors included trial and block order. The fixed experimental predictors were SOA, S1 type, relatedness, and all interactions. The random effects structure included random intercepts by participant and item, and random slopes of SOA, S1 type and relatedness by participant and item.

For the control predictors, the effect of trial just failed to reach significance (estimate $=-2.67 \mathrm{e}-05, \mathrm{SE}=1.36 \mathrm{e}-05, t=-1.97, \mathrm{CI}$ [-5.3e-05 -8.8e-07]). We found no main effect of block order $(\mathrm{t}<2)$.

For the experimental predictors, we found a main effect of SOA (estimate $=6.79 \mathrm{e}-02, \mathrm{SE}=2.58 \mathrm{e}-03, t=26.31$, CI $[0.0630 .073]$ ) as responses at $0 \mathrm{~ms}$ were slower than at $1000 \mathrm{~ms}$, a main effect of relatedness (estimate $=3.82 \mathrm{e}-03, \mathrm{SE}=1.32 \mathrm{e}-03, t=2.9$, CI $[0.0010 .006]$ ) as latencies in the related condition were slower than in the unrelated condition, and no main effect of S1 type (estimate $=4.1 \mathrm{e}-03, \mathrm{SE}=$ $2.47 \mathrm{e}-03, t=1.66$, CI $[-0.00080 .009]$ ). The interaction between SOA and $\mathrm{S} 1$ type was significant (estimate $=6.56 \mathrm{e}-03, \mathrm{SE}=1.02 \mathrm{e}-03$, $t=6.46$, CI [0.005 0.009]), driven by the fact that at $0 \mathrm{~ms}$, latencies with $\mathrm{S} 1$ syllables were $60 \mathrm{~ms}$ slower than with $\mathrm{S} 1$ tones $\left(M_{\text {syllable }}=\right.$ $\left.1154 \mathrm{~ms}, \mathrm{M}_{\text {tone }}=1094 \mathrm{~ms} ; \mathrm{t}(49.5)=4, p<.001\right)$, whereas at $1000 \mathrm{~ms}$

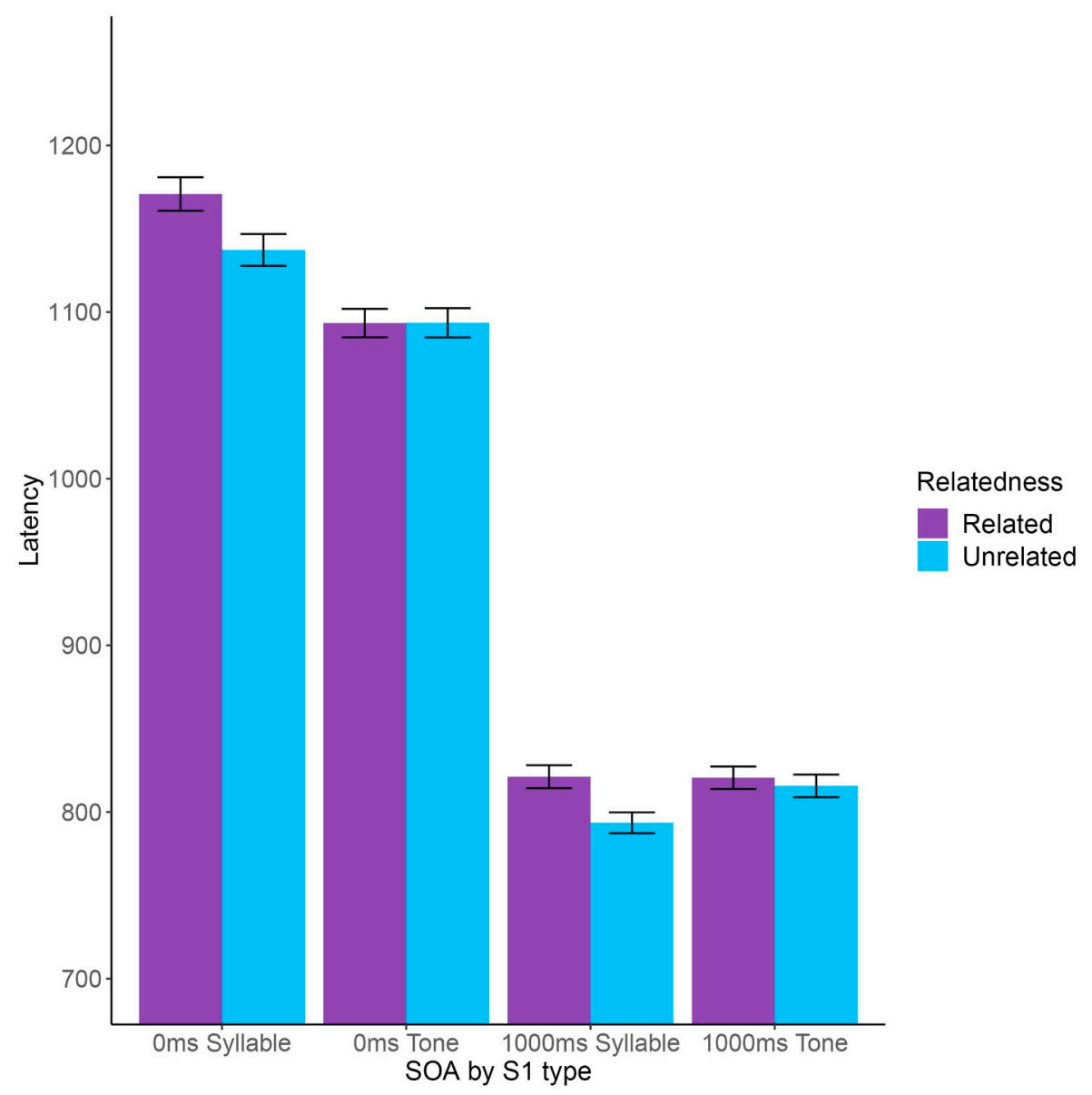

Fig. 4. Experiment 2: Mean naming latencies (in ms) by S1 type, SOA and relatedness. Error bars are standard errors and are calculated within-participant. Note that the $y$-axis does not begin at zero. 
Table 3

Experiment 2: Error proportions in naming latencies by S1 type, SOA and relatedness.

\begin{tabular}{llllll}
\hline & \multicolumn{2}{l}{ S1 syllable } & & & \multicolumn{2}{l}{ S1 tone } \\
\cline { 2 - 3 } \cline { 5 - 6 } & SOA 0 ms & SOA 1000 ms & & SOA 0 ms & SOA 1000 ms \\
\hline Related & 0.09 & 0.1 & & 0.06 & 0.11 \\
Unrelated & 0.07 & 0.09 & & 0.06 & 0.08 \\
\hline
\end{tabular}

latencies were more similar (11 ms difference; $\mathrm{M}_{\text {syllable }}=807 \mathrm{~ms}$, $\left.\mathrm{M}_{\text {tone }}=818 \mathrm{~ms} ; \mathrm{t}(50.4)=-0.9, p=.36\right)$. Relatedness and S1 type also interacted (estimate $=2.85 \mathrm{e}-03, \mathrm{SE}=1.02 \mathrm{e}-03, t=2.8$, CI $[0.0009$ $0.005])$, demonstrating that the relatedness effect with $S 1$ syllables was significantly larger $(\mathrm{t}(64.03)=4, p<.001)$ than with $\mathrm{S} 1$ tones $(\mathrm{t}$ $(62.47)=0.59, p=.56)$. SOA and relatedness did not interact $(\mathrm{t}<1)$, meaning that the semantic interference effect was the same size at both SOAs. The three-way interaction also did not reach significance $(\mathrm{t}<1)$.

$8.4 \%$ of the naming data were analysable naming task errors, presented in Table 3. A mixed effects model of the error data was conducted with a similar model structure as for the naming latency data, but included a random slope of SOA by item. There was a main effect of SOA (estimate $=0.19, \mathrm{SE}=0.05, \mathrm{z}=4.04, p<.001$ ), as participants made more errors at SOA $1000 \mathrm{~ms}$ than at SOA $0 \mathrm{~ms}$. There was a main effect of relatedness (estimate $=-0.15, \mathrm{SE}=0.06, \mathrm{z}=-2.26$, $p=.02$ ), as more errors were made in the related than the unrelated condition. There was main effect of $S 1$ type (estimate $=-0.15$, $\mathrm{SE}=0.05, \mathrm{z}=-2.87, p=.004)$, as more errors were made with $\mathrm{S} 1$ syllables than S1 tones. The interaction between SOA and S1 type was significant (estimate $=-0.09, \mathrm{SE}=0.04, \mathrm{z}=-2.12, p=.03$ ), as there were more errors with S1 syllables at SOA $0 \mathrm{~ms}$ than with S1 tones $(\mathrm{z}=-3.28, p=.001)$, with similar error proportions at SOA $1000 \mathrm{~ms}$ $(\mathrm{z}=-1.09, p=.27)$. The error data is in line with the latency data, and there is no evidence for a speed-accuracy trade-off.

\subsubsection{Experiment 2: Reading latencies}

Fig. 5 shows the mean reading latencies by S1 type, SOA, and relatedness. The descriptive relatedness effect at SOA $0 \mathrm{~ms}$ with $\mathrm{S} 1$ syllables was $-2 \mathrm{~ms}$ and with S1 tones was $-2 \mathrm{~ms}$. At SOA $1000 \mathrm{~ms}$ the effect with S1 syllables was $-3 \mathrm{~ms}$ and with $\mathrm{S} 1$ tones was $-8 \mathrm{~ms}$.

A linear mixed effects model included log transformed reading latencies as the dependent variable. Fixed control predictors included trial and block order. The fixed experimental predictors were SOA, S1 type, relatedness, and all interactions. The random effects structure included random intercepts by participant and item, random slopes of SOA and S1 type by participant and item, and a random slope of relatedness by participant.

We found no main effects of trial or block order (both t's $<1$ ). There was a significant effect of SOA (estimate $=0.11, \mathrm{SE}=3.06 \mathrm{e}-03$, $t=36.01$, CI [ 0.1040 .116$]$ ), as latencies were longer at SOA $0 \mathrm{~ms}$ than at SOA $1000 \mathrm{~ms}$, and a significant effect ofS1 type (estimate $=7.12 \mathrm{e}$ $03, \mathrm{SE}=2.57 \mathrm{e}-03, t=2.77, \mathrm{CI}[0.0020 .012]$ ), as latencies were longer with S1 syllables compared to S1 tones. There was no main effect of relatedness $(t<2)$. The only interaction to reach significance was the SOA by $\mathrm{S} 1$ type interaction (estimate $=7.31 \mathrm{e}-03, \mathrm{SE}=1.03 \mathrm{e}-04$, $t=7.09$, CI [0.005 0.093]; all other interactions $t<1$ ). This was because reading latencies at $0 \mathrm{~ms}$ with $\mathrm{S} 1$ syllables were $65 \mathrm{~ms}$ longer

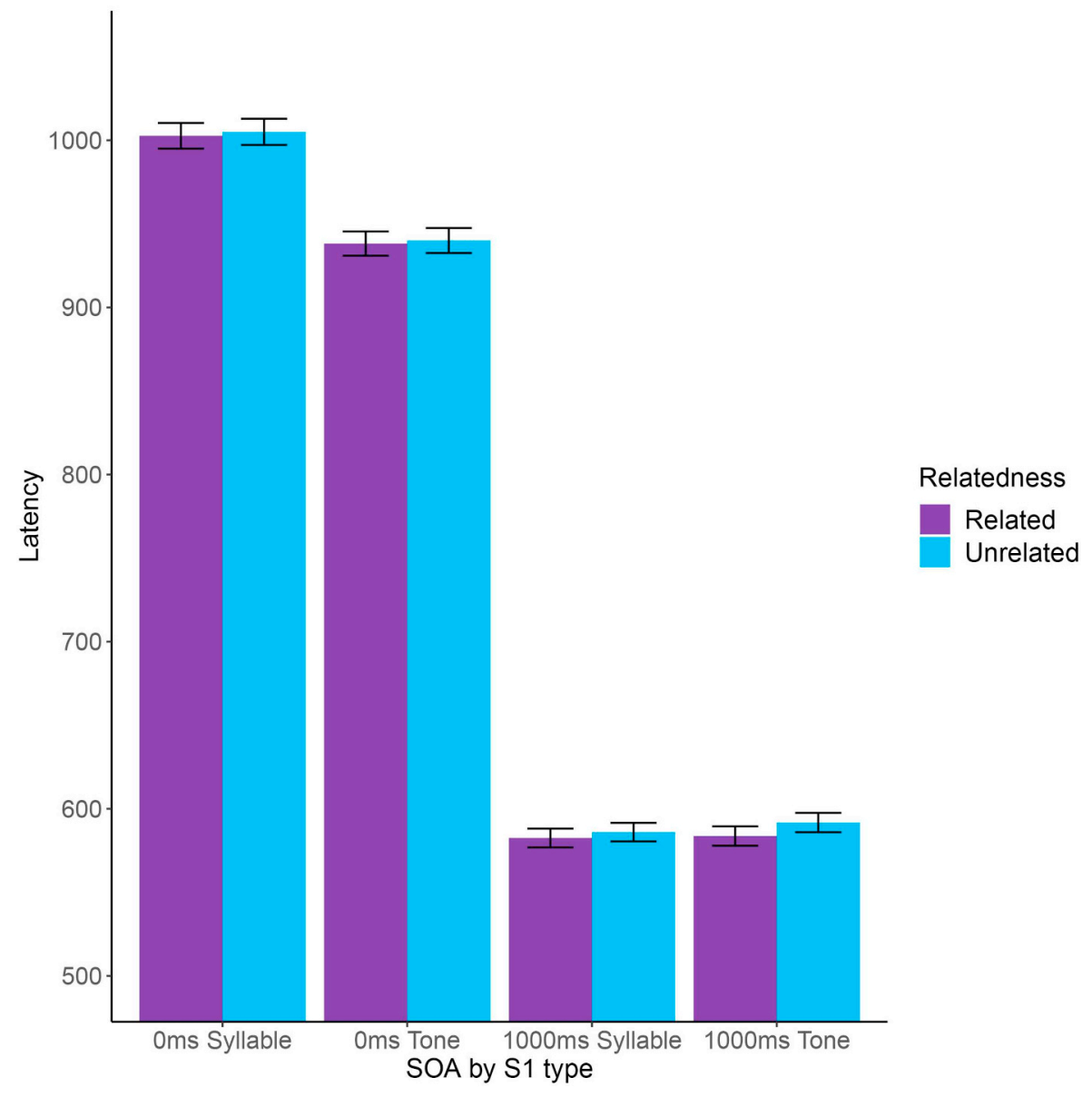

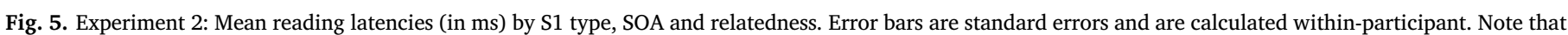
the $y$-axis does not begin at zero. 
Table 4

Experiment 2: Error proportions in reading latencies by S1 type, SOA and relatedness.

\begin{tabular}{llllll}
\hline & \multicolumn{2}{l}{ S1 syllable } & & & \multicolumn{2}{l}{ S1 tone } \\
\cline { 2 - 3 } \cline { 5 - 6 } & SOA 0 ms & SOA 1000 ms & & SOA 0 ms & SOA 1000 ms \\
\hline Related & 0.03 & 0.02 & & 0.03 & 0.03 \\
Unrelated & 0.02 & 0.02 & & 0.02 & 0.02 \\
\hline
\end{tabular}

than with $\mathrm{S} 1$ tones $\left(\mathrm{M}_{\text {syllable }}=1004 \mathrm{~ms}, \mathrm{M}_{\text {tone }}=939 \mathrm{~ms} ; \mathrm{t}(44.81)=\right.$ $5.23, p<.0001$ ), whereas at $1000 \mathrm{~ms}$ the latencies were roughly equal $\left(\mathrm{M}_{\text {syllable }}=584 \mathrm{~ms}, \mathrm{M}_{\text {tone }}=588 \mathrm{~ms} ; \mathrm{t}(44.78)=-0.07, p=.95\right)$.

$2.4 \%$ of the reading data were analysable errors, presented in Table 4. A mixed effects model of the error data was conducted with a similar model structure as for the reading latency data, but with no random slope of SOA by participant, and no random slopes by item. There were no significant effects.

\subsection{Discussion}

For the picture naming latencies, we obtained a main effect of SOA, indicating that the participants named the pictures faster when the cue to do so preceded rather than coincided with picture onset. There was also a main effect of relatedness, showing that related distractor words interfered more with picture naming than unrelated ones. Importantly, these effects did not interact. Thus, there was no evidence that at the $0 \mathrm{~ms} \mathrm{SOA}$, the interference effect was absorbed by the cognitive slack created by the response choice task. This may mean that the response choice task was made too fast to create sufficient slack. Alternatively, it may mean that the participants scheduled the tasks differently: Instead of selecting the picture name before making the task choice, as Piai et al. (2015) proposed, participants first selected the task and then proceeded to select the picture name.

There was no triple interaction of SOA, relatedness and S1 type, indicating that the way the response choice task and picture naming were coordinated was not affected by the linguistic or non-linguistic nature of S1. We did, however, find an interaction of S1 type with SOA, showing that at the SOA of $0 \mathrm{~ms}$, but not at the SOA of $1000 \mathrm{~ms}$, syllables interfered more with picture naming than tones. The same pattern had also been observed in Experiment 1. This interaction was also seen for the reading times, along with a main effect of SOA. Thus, both tasks - picture naming and reading - were hindered more by the presentation of the syllables compared to the tones.

While these results form a coherent pattern, suggesting sequential processing of the two stimuli and little influence of S1 type on the processing strategy, there was one important unexpected finding, namely the interaction of relatedness and S1 type. As Fig. 4 shows, the semantic interference effect was obtained at both SOAs when S1 was a syllable, but it was not seen at either SOA when S1 was a tone. While the absence of a distractor effect at the $0 \mathrm{~ms}$ SOA is consistent with the results obtained by Piai et al. (2015), its absence at the $1000 \mathrm{~ms}$ SOA is surprising and complicates the interpretation of the remaining findings of this experiment. This is because effects at the $0 \mathrm{~ms}$ SOA are interpreted by comparison with 'baseline' effects at the $1000 \mathrm{~ms}$ SOA.

Our TC experiment differed from the experiments by Piai et al. (2015) in a number of ways. One potentially important difference is that we varied SOA within blocks (as in our Experiment 1), whereas Piai et al. (2015) used a blocked design. We opted for within-block variation of SOA because our experiments featured an additional variable, S1 type, which was varied between blocks. Both within and between-block manipulations of SOA have been used in earlier TC studies (for within-block manipulations see Besner \& Care, 2003; Besner \& Risko, 2005; O'Malley \& Besner, 2011; Paulitzki, Risko, O'Malley, Stolz, \& Besner, 2009; Risko \& Besner, 2008; for between-block (but also between-participant) manipulations see Janssen et al., 2008; Mädebach et al., 2011; Piai et al., 2011). From this literature it is not obvious how this design choice would affect the strength of the semantic interference effect. However, in order to facilitate the comparison of the present results to those obtained by Piai et al. (2015), we conducted an additional experiment varying SOA between rather than within blocks.

\section{Experiment 3: Blocked task choice paradigm}

Experiment 3 was the same in design and materials to Experiment 2 except that SOA was blocked rather than variable.

\subsection{Methodology}

\subsubsection{Participants}

38 participants $(M=21$ years, $S D=2.9,30$ female) were recruited from the Max Planck Participant Database. All self-reported as righthanded, with no language, sight, or hearing disorders. Participants were paid $€ 12$ for participation, and were given sweets to motivate them to stay on task. The experiment was granted ethical approval by the Radboud University Social Sciences ethics committee in accordance with the Declaration of Helsinki.

\subsubsection{Materials, Apparatus \& Procedure}

The same materials, apparatus, and procedure were used as in Experiment 2.

\subsubsection{Design}

The design was the same as for Experiment 2, except that SOA was blocked. Block order was counterbalanced such that a participant would either carry out blocks in the order $0 \mathrm{~ms}-1000 \mathrm{~ms}$, or $1000 \mathrm{~ms}-$ $0 \mathrm{~ms}$. The same block order was used for both $\mathrm{S} 1$ types (e.g., participant 1 carried out the S1 syllable 0 ms block, then the S1 syllable $1000 \mathrm{~ms}$ block, then the $\mathrm{S} 1$ tone $0 \mathrm{~ms}$ block, then the $\mathrm{S} 1$ tone $1000 \mathrm{~ms}$ block).

\subsection{Results}

\subsubsection{Pre-processing and analysis}

Data were checked for errors in the same way and with the same error criteria as in Experiments 1 and 2. One participant was removed ( $>20 \%$ errors), leaving analysable datasets from 37 participants. Speech latencies were also preprocessed in the same way. 6.5\% $(\mathrm{N}=1231)$ data was removed due to errors. Data were analysed and are presented in the same way as Experiment 2.

\subsubsection{Experiment 3: Naming latencies}

Naming latencies for Experiment 3 are presented in Fig. 6. At SOA $0 \mathrm{~ms}$, there was a descriptive interference effect of $20 \mathrm{~ms}$ with S1 syllables and of $18 \mathrm{~ms}$ in with S1 tones. At SOA $1000 \mathrm{~ms}$, there was a descriptive interference effect of $24 \mathrm{~ms}$ with S1 syllables and of $15 \mathrm{~ms}$ with S1 tones.

A linear mixed effects model included log transformed naming latencies as the dependent variable. Fixed control predictors included trial and block order. The fixed experimental predictors were SOA, S1 type, relatedness, and all interactions. The random effects structure included random intercepts by participant and item, random slopes of S1 type and relatedness by participant and item, and a random slope of SOA by participant.

For the control predictors trial and order, there were no significant main effects $(t<2)$. For the experimental predictors, we found main effects of SOA (estimate $=5.24 \mathrm{e}-02, \mathrm{SE}=3.1 \mathrm{e}-03, t=16.9$, CI $[0.046$ $0.058]$ ), as latencies were longer at SOA $0 \mathrm{~ms}$ than SOA $1000 \mathrm{~ms}$, and relatedness (estimate $=4.77 \mathrm{e}-03, \mathrm{SE}=1.64 \mathrm{e}-03, t=2.9$, CI $[0.002$ $0.008]$ ), as latencies were longer in the related condition than the unrelated condition. There was no effect of $S 1$ type (estimate $=3.39 \mathrm{e}-03$, $\mathrm{SE}=2.6 \mathrm{e}-03, t=1.3$, CI $[-0.0020 .009])$, meaning that latencies were similar regardless of S1 type. The interaction between S1 type and SOA 


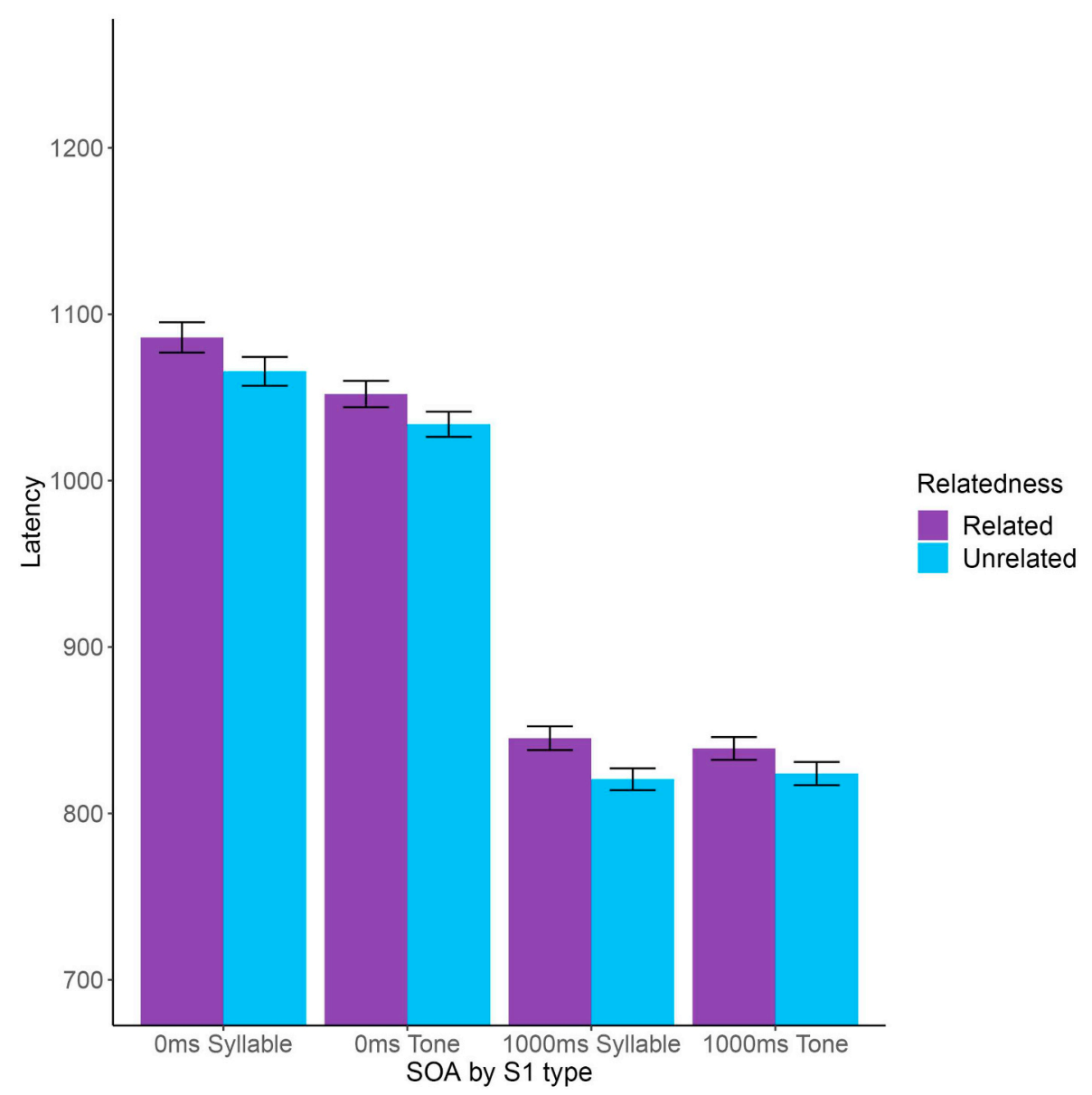

Fig. 6. Experiment 3: Mean naming latencies (in ms) by S1 type, SOA and relatedness. Error bars are standard error and are calculated within-participant. Note that the $y$-axis does not begin at zero.

was significant (estimate $=3.15 \mathrm{e}-03, \mathrm{SE}=9.7 \mathrm{e}-04, t=3.2$, CI $[0.001$ 0.005]), driven by the fact that at SOA $0 \mathrm{~ms}$, latencies with $\mathrm{S} 1$ syllables were $33 \mathrm{~ms}$ slower than latencies with $\mathrm{S} 1$ tones $\left(\mathrm{M}_{\text {syllable }}\right.$ $=1076 \mathrm{~ms}, \mathrm{M}_{\text {tone }}=1043 \mathrm{~ms} ; \mathrm{t}(48.39)=2.35, p=.02$ ), whereas at SOA $1000 \mathrm{~ms}$ the difference was smaller at $2 \mathrm{~ms}\left(\mathrm{M}_{\text {syllable }}=833 \mathrm{~ms}, \mathrm{M}_{\text {tone }}=\right.$ $831 \mathrm{~ms} ; \mathrm{t}(47.86)=0.08, p=.93)$. All other interactions were not significant $(\mathrm{t}<1)$. The lack of significant interactions involving relatedness indicates that the relatedness effect did not vary by $\mathrm{S} 1$ type or by SOA.

$5.6 \%$ of the naming data were analysable errors, presented in Table 5. A mixed effects model of the error data was conducted with a similar model structure as for the naming latency data, but with no random slopes by item. There was a main effect of SOA (estimate $=-0.21, \mathrm{SE}=0.07, \mathrm{z}=-3, p=.003$ ), as there were more errors at SOA $0 \mathrm{~ms}$ than SOA $1000 \mathrm{~ms}$. There was a main effect of S1 type (estimate $=0.16, \mathrm{SE}=0.08, \mathrm{z}=-2.18, p=.03$ ), as there were more errors with S1 syllables. The interaction between SOA and S1 type was significant (estimate $=-0.13, \mathrm{SE}=0.05, \mathrm{z}=-2.55, p=.01$ ), as there were more errors with S1 syllables than S1 tones at SOA $0 \mathrm{~ms}$

Table 5

Experiment 3: Error proportions in naming latencies by $\mathrm{S} 1$ type, SOA and relatedness.

\begin{tabular}{llllll}
\hline & \multicolumn{2}{l}{ S1 syllable } & & & \multicolumn{2}{l}{ S1 tone } & \\
\cline { 2 - 3 } \cline { 5 - 6 } & SOA 0 ms & SOA 1000 ms & & SOA 0 ms & SOA 1000 ms \\
\hline Related & 0.1 & 0.05 & & 0.06 & 0.05 \\
Unrelated & 0.07 & 0.04 & & 0.04 & 0.05 \\
\hline
\end{tabular}

$(\mathrm{z}=-3.15, p=.002)$, whereas the difference at SOA $1000 \mathrm{~ms}$ was much smaller $(\mathrm{z}=-0.41, p=.68)$. There is no evidence for a speedaccuracy trade-off.

\subsubsection{Experiment 3: Reading latencies}

Reading latencies are presented in Fig. 7. At SOA $0 \mathrm{~ms}$, there was a descriptive interference effect of $5 \mathrm{~ms}$ with $\mathrm{S} 1$ syllables and of $5 \mathrm{~ms}$ with S1 tones. At SOA $1000 \mathrm{~ms}$, there was a descriptive interference effect of $5 \mathrm{~ms}$ with $\mathrm{S} 1$ syllables and of $-5 \mathrm{~ms}$ with $\mathrm{S} 1$ tones.

A linear mixed effects model included log transformed reading latencies as the dependent variable. Fixed control predictors included trial and block order. The fixed experimental predictors were SOA, S1 type, relatedness, and all interactions. The random effects structure included random intercepts by participant and item, and random slopes of SOA, S1 type and relatedness by participant and item.

For the control predictors, there was a main effect of trial (estimate $=5.92 \mathrm{e}-05, \mathrm{SE}=2.63 \mathrm{e}-05, t=2.26$, CI [7.75e-06 1.11e-04]), as participants slowed down within each block of the experiment. There was no effect of block order $(t<1)$. For the experimental predictors, we found a main effect of SOA $(9.34 \mathrm{e}-02$, SE $=3.83 \mathrm{e}-03, t=24.38$, CI [0.086 0.1]), as participants read aloud faster at the $1000 \mathrm{~ms}$ SOA than the $0 \mathrm{~ms}$ SOA. There were no main effects of $\mathrm{S} 1$ type or relatedness $(\mathrm{t}$ 's $<2$ ). None of the interactions reached significance (all t's $<2$ ).

$1.9 \%$ of the reading data were analysable errors, presented in Table 6. A mixed effects model of the error data was conducted with a similar model structure as for the reading latency data, but with no random slopes of SOA or relatedness by participant or by item. There was a main effect of trial (estimate $=0.24, S E=0.08, z=3.19$, $p=.001$ ) as participants made more errors as the experiment went on. 


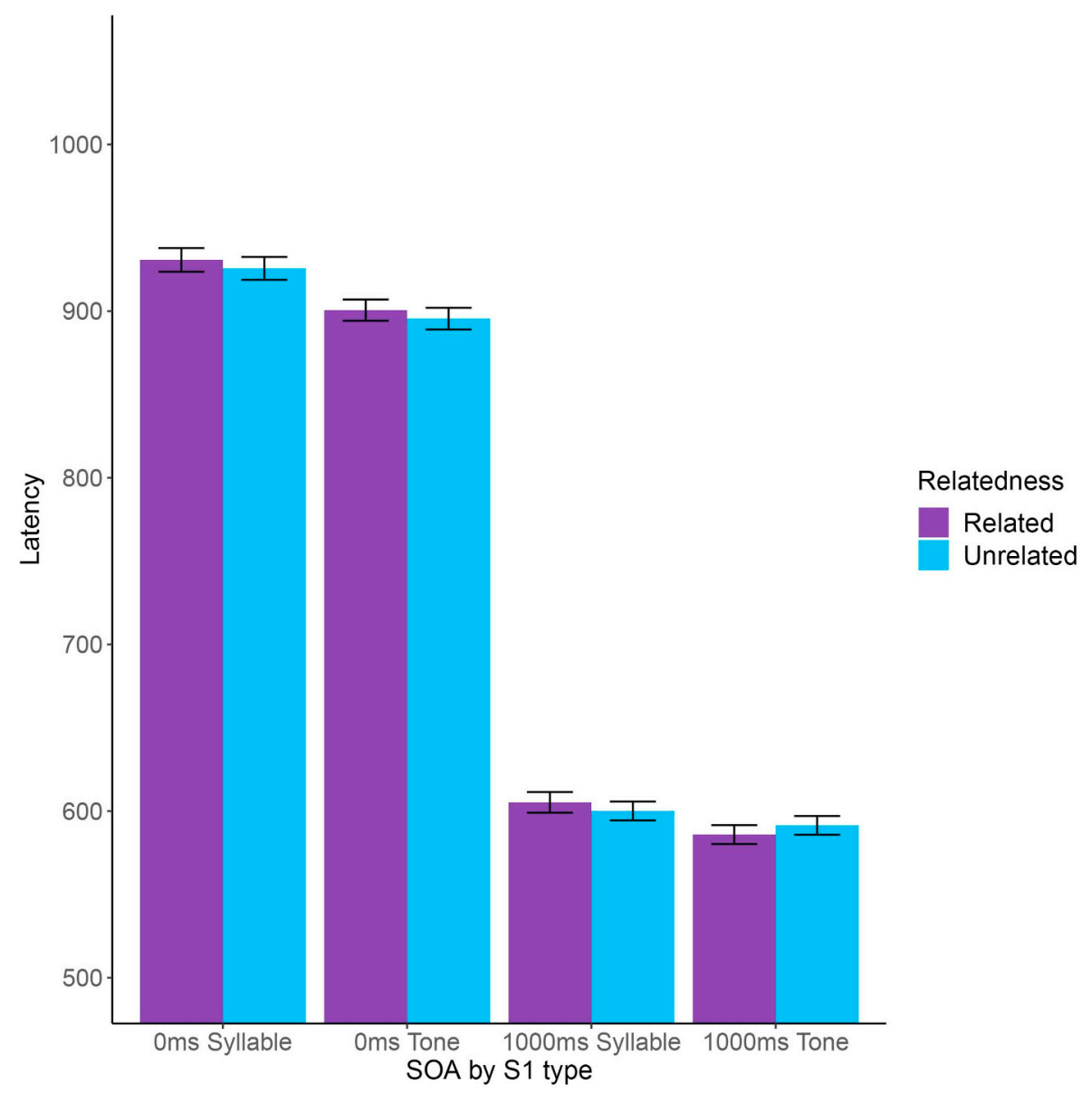

Fig. 7. Experiment 3: Mean reading latencies (in ms) by S1 type, SOA and relatedness. Error bars are standard error and are calculated within-participant. Note that the $y$-axis does not begin at zero.

Table 6

Experiment 3: Error proportions in reading latencies by S1 type, SOA and relatedness.

\begin{tabular}{llllll}
\hline & \multicolumn{2}{l}{ S1 syllable } & & & \multicolumn{2}{l}{ S1 tone } \\
\cline { 2 - 3 } \cline { 5 - 6 } \cline { 5 - 6 } & SOA 0 ms & SOA 1000 ms & & SOA 0 ms & SOA 1000 ms \\
\hline Related & 0.03 & 0.01 & & 0.02 & 0.01 \\
Unrelated & 0.03 & 0.01 & & 0.02 & 0.02 \\
\hline
\end{tabular}

There was a main effect of SOA (estimate $=-0.36, \mathrm{SE}=0.08$, $\mathrm{z}=-4.45, p<.001$ ), as there were more errors at SOA $0 \mathrm{~ms}$ than at SOA $1000 \mathrm{~ms}$. No other effects were significant. There is no evidence for a speed-accuracy trade-off.

\subsection{Discussion}

The results of Experiment 3 largely correspond to those of Experiment 2. For the picture naming latencies, we obtained the expected effects of SOA and relatedness, with picture naming being faster at the $1000 \mathrm{~ms}$ than the $0 \mathrm{~ms} \mathrm{SOA}$, and faster with an unrelated distractor compared to related distractor. These effects did not interact, which, as explained above, may either indicate that at the $0 \mathrm{~ms}$ SOA the processing of the auditory stimulus did not create enough slack to absorb the semantic interference effect, or that participants strategically scheduled lexical selection to follow, rather than precede, the choice of task. Importantly, the interaction of relatedness and S1 type, seen in Experiment 2, was not significant. Instead, moderate semantic interference effects were seen in naming for both $\mathrm{S} 1$ types and at both SOAs.
As before, S1 type interacted with SOA, with syllables interfering more with picture naming than tones, but only at the short SOA.

For the reading latencies, we only observed the expected main effect of SOA, with reading times being shorter at the $0 \mathrm{~ms}$ SOA than at the $1000 \mathrm{~ms}$ SOA. Thus, both responses, picture naming and word reading, were initiated faster when the cue preceded the picture compared to when it occurred at the same time, as one would expect. The S1 type effect on reading RTs at the $0 \mathrm{~ms}$ SOA, seen in Experiment 2, was not replicated.

\section{Bayesian analysis of RTs}

Given the inconsistency of the patterns of results obtained in our experiments and the TC experiment reported by Piai et al. (2015), we explored whether the experiments were adequately powered to draw conclusions regarding the presence or absence of the semantic interference effect. If lexical selection occurred in parallel with the processing of S1, most participants should show negligible semantic interference effects. We considered effects (absolute response time differences between the related and unrelated condition) of $|10| \mathrm{ms}$ to be negligible. Most published semantic interference experiments obtained effect sizes of at least $20 \mathrm{~ms}$ (Ayora et al., 2011; Damian \& Martin, 1999; Ferreira \& Pashler, 2002; Glaser \& Düngelhoff, 1984; Piai et al., 2011, 2014; Schnur \& Martin, 2012; Schriefers et al., 1990), and in the present experiments, the smallest significant interference effect was $15 \mathrm{~ms}$. Thus, a $10 \mathrm{~ms}$ band seemed a good range for negligible effects.

The panels in Figs. 8 and 9 display the semantic interference effect by participant at SOA $0 \mathrm{~ms}$ in each experiment. The black dotted lines at zero make it easier to see positive and negative effects. The grey band 


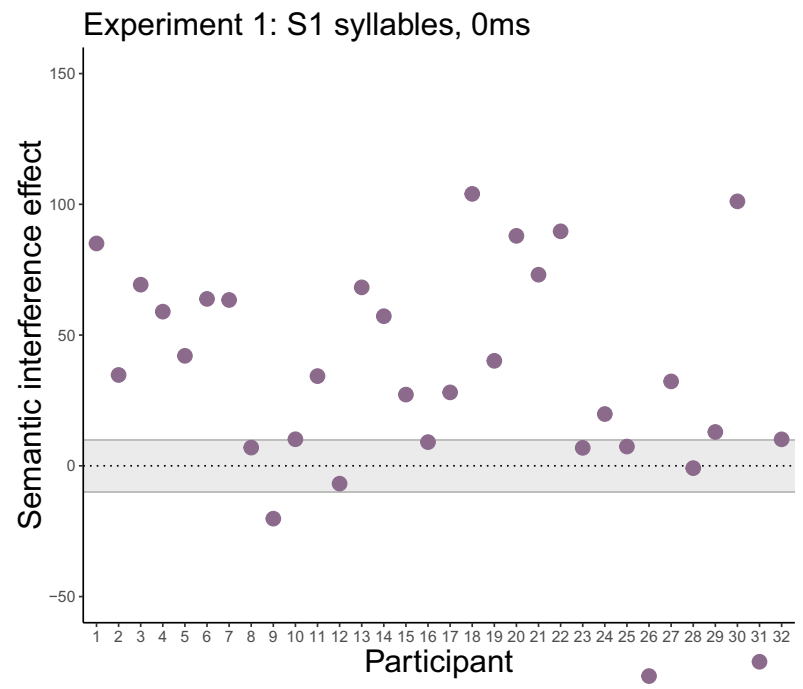

Experiment 2: S1 syllables, 0ms

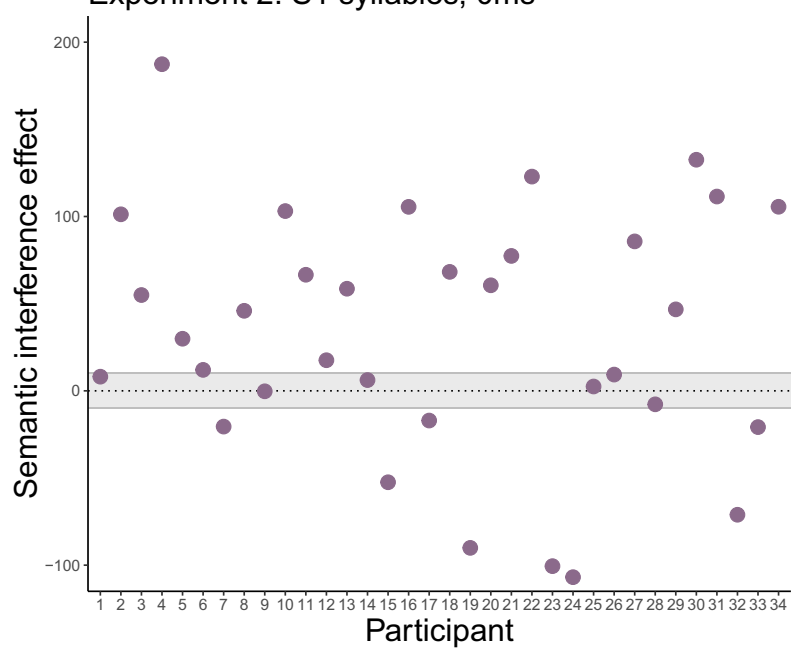

Experiment 3: S1 syllables, 0ms

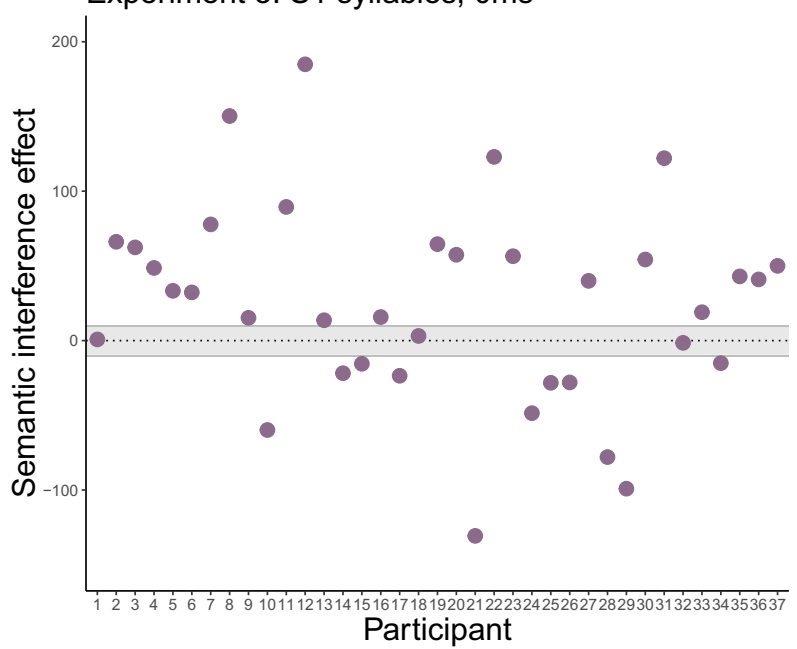

Experiment 1: $\mathrm{S} 1$ tones, 0ms

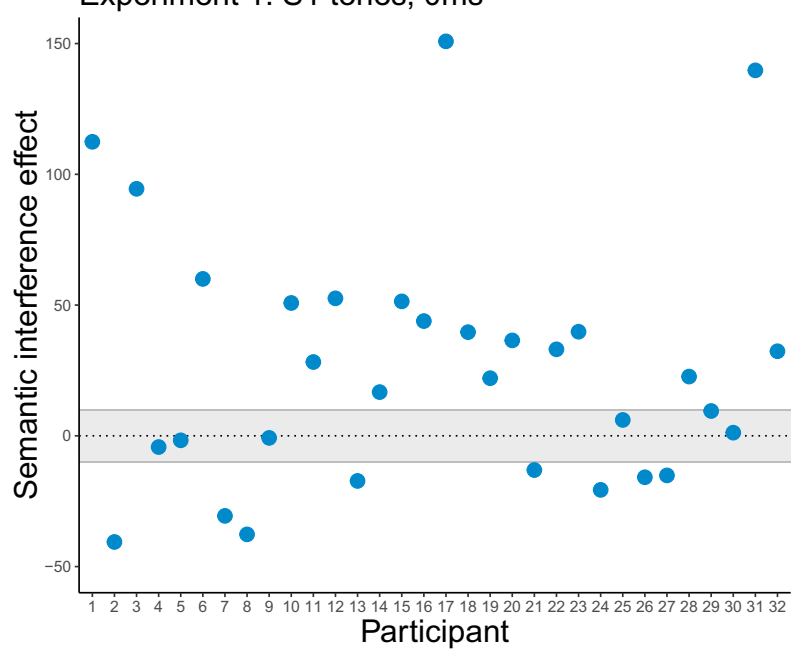

Experiment 2: $\mathrm{S} 1$ tones, $0 \mathrm{~ms}$

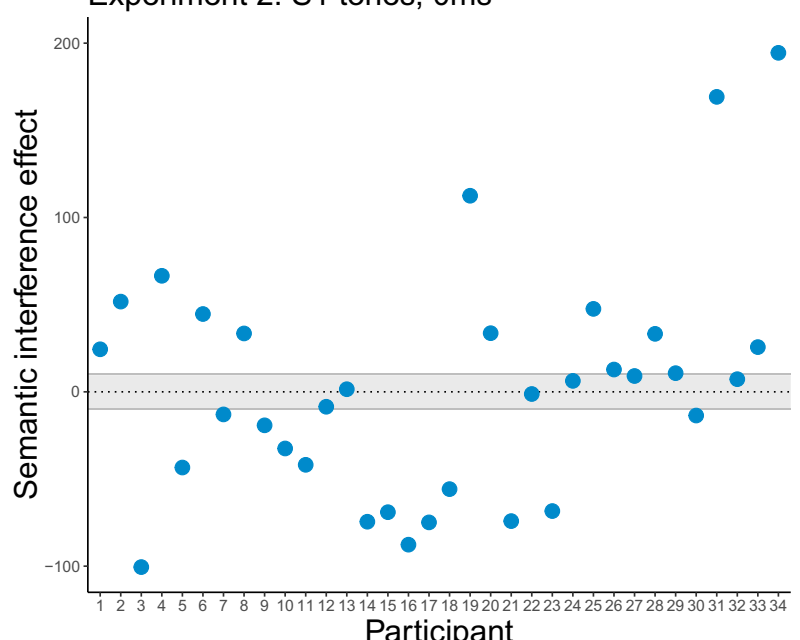

Experiment 3: $\mathrm{S} 1$ tones, $0 \mathrm{~ms}$

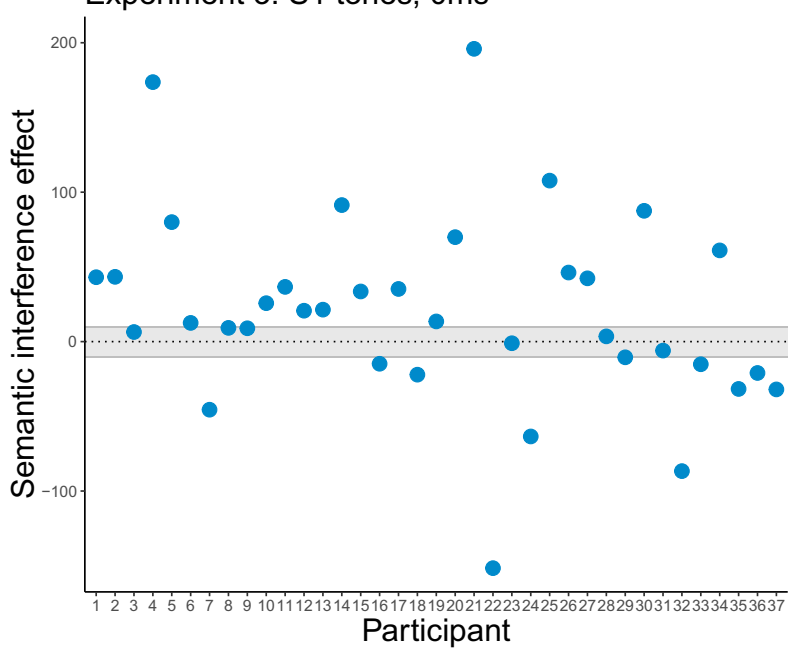

Fig. 8. Semantic interference effects (in ms) by participant in Experiments 1,2 and 3 at the $0 \mathrm{~ms}$ SOA only. The left column of figures (with purple dots) plots S1 syllables in each experiment. The right column (with blue dots) plots $\mathrm{S} 1$ tones. Top row $=$ Experiment 1 ; middle row $=$ Experiment 2 ; bottom row $=$ Experiment 3 . A dotted line is plotted at $\mathrm{y}=0 \mathrm{~ms}$ for ease of interpreting positive and negative effects. The grey band spans $\mathrm{y}=10 \mathrm{~ms}$ to $\mathrm{y}=-10 \mathrm{~ms}$ and signifies the band of negligible effects. (For interpretation of the references to colour in this figure legend, the reader is referred to the web version of this article.) 

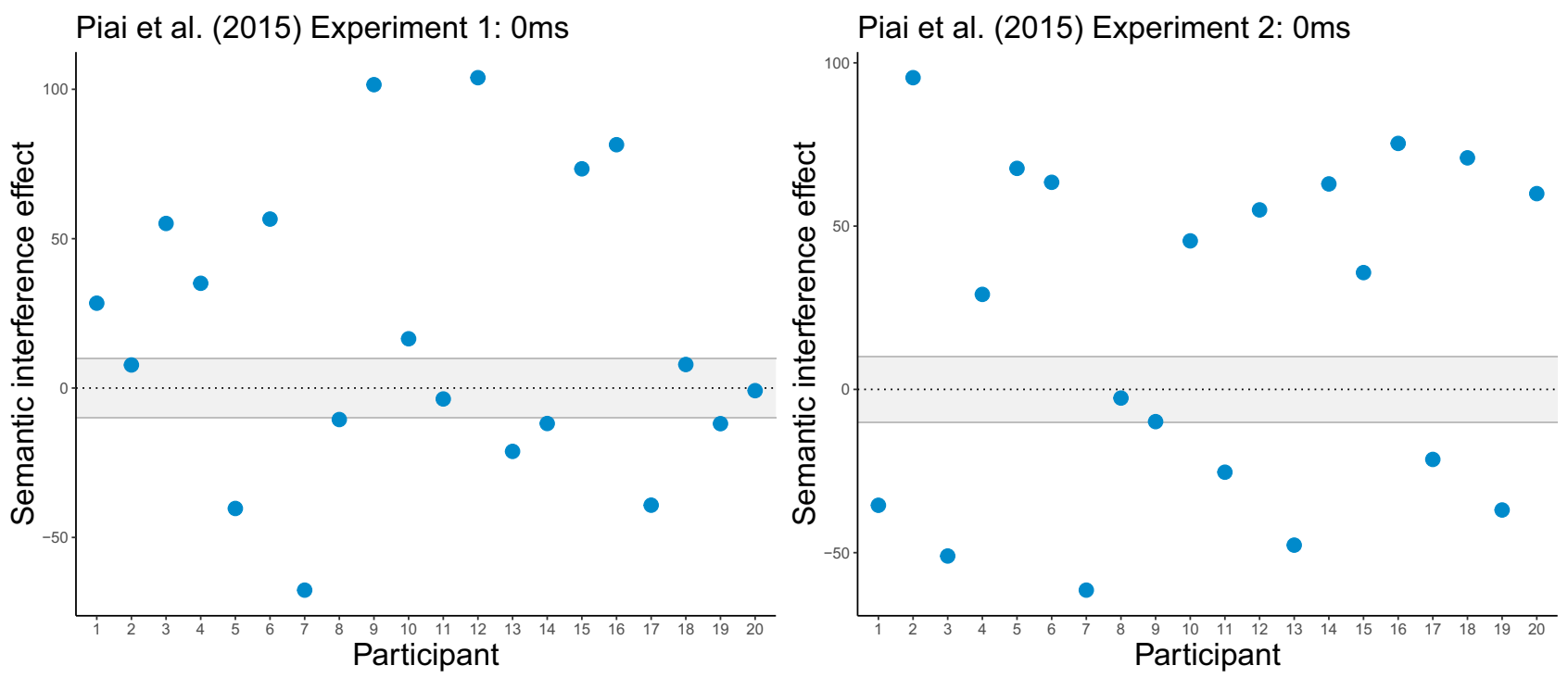

Fig. 9. Semantic interference effects (in ms) by participant in Experiments 1 (left) and 2 (right) from Piai et al. (2015) at the 0 ms SOA only. A dotted line is plotted where $\mathrm{y}=0 \mathrm{~ms}$ for ease of interpreting positive and negative effects. The grey band spans $\mathrm{y}=10 \mathrm{~ms}$ to $\mathrm{y}=-10 \mathrm{~ms}$ and signifies the negligible band of effects.

spans $10 \mathrm{~ms}$ to $-10 \mathrm{~ms}$ to show which effects fall within this negligible band. In the top row of Fig. 8, showing the results of Experiment 1, most participants have positive semantic interference effects with both S1 types. In the middle row (Experiment 2), with S1 syllables (left middle figure), the interference effects are still largely positive, but there is a wider distribution than in Experiment 1. With S1 tones (middle right figure), the interference effects are almost equally balanced between positive and negative. In the bottom row (Experiment 3), with both S1 types the effect distributions are wide, but most are positive. In Experiments 1 and 2 reported in Piai et al. (2015), displayed in Fig. 9, almost equal numbers of participants have positive and negative interference effects. Across all experiments, very few individual semantic interference effects are in the negligible band (8-25\% of effects by experiment). Thus, from visual inspection of the effect patterns, we conclude that there is no evidence supporting parallel processing.

We carried out a series of Bayesian paired $t$-tests to test whether Piai et al. (2015) and the present experiments had enough evidence to support the presence or absence of a semantic interference effect. Bayesian two-tailed $t$-tests carried out in $\mathrm{R}$ compared the related and unrelated naming latencies at each SOA with each S1 type using the BEST package (Kruschke \& Meredith, 2017), and Bayes Factors were calculated using the BayesFactor package (Morey \& Rouder, 2015). For each $t$-test three chains with approximately 34,000 iterations per chain were used for calculation. One chain with 34,000 iterations was used for calculation of each Bayes Factor. We set a prior distribution as the log-transformed effect size of $24 \mathrm{~ms}$ (the average of the 16 semantic interference effects found in the three experiments reported in this paper and the two experiments reported in Piai et al., 2015), with a standard deviation of $8 \mathrm{~ms}$. When using the default non-informative prior, the same pattern of results was found. All results are presented in Table 7. The column Difference reports the difference between conditions on the log (base 10) scale. The column 95\% HDI reports the highest density credible interval. This interval contains the $95 \%$ most plausible values of the effect size. The column $\%$ below 0 shows the percentage of the effect sizes which fall below zero. The \% in ROPE column reports what proportion of the data falls within a region of practical equivalence (ROPE; Kruschke, Aguinis, \& Joo, 2012). We set the region of practical equivalence to be approximately $10 \mathrm{~ms}$, as displayed in Figs. 8 and 9. Thus, this column tells us what proportion of the difference between related and unrelated conditions is essentially negligible. The final column, $B F$, displays the Bayes Factor for the comparison. Following Kass and Raftery (1995) we take Bayes
Factors $>3$ as indicating support for the alternate hypothesis, as $>10$ providing strong support for the alternate hypothesis, and $<1 / 3$ (0.334) as providing support for the null hypothesis.

For Experiment 1 we have fairly strong support at both SOAs with both $\mathrm{S} 1$ types for the presence of a semantic interference effect. With S1 tones at $0 \mathrm{~ms}$ we found slightly weaker support. Almost no data in this experiment fell below zero. Additionally, almost no data fell within the ROPE, meaning that at least $97 \%$ of the effects are larger than a negligible effect.

In Experiment 2 we found evidence for the presence of a semantic interference effect with S1 syllables at SOA $1000 \mathrm{~ms}$, and inconclusive evidence at SOA $0 \mathrm{~ms}$. However, only a small proportion of data fell below zero $(<1 \%)$ and only $2 \%$ of the effect fell within the negligible band. This means that the effect is largely meaningful. For S1 tones, we found evidence supporting a null effect, meaning that in this condition the absence of the semantic interference effect is meaningful. The Bayes Factors for tests at the two SOAs were both lower than 0.334, supporting the null hypothesis. The HDI for both tests ranged from negative to positive, and almost $50 \%$ of the data at $0 \mathrm{~ms}$ was below zero. Around half of the data from both OAs fell within the ROPE, meaning that approximately half of the effects were within the negligible band. Therefore, we find support for the null hypothesis.

In Experiment 3 we do not have enough evidence to support either the null or the alternate hypothesis in either condition at the early SOAs, and at the late SOA with S1 tones. The Bayes Factors were inconclusive, and almost $10 \%$ of the effects fell within the negligible band. The HDIs were also very wide, estimating a wide range of the size of the plausible effect. Only with S1 syllables at $1000 \mathrm{~ms}$ did we find evidence, albeit weak, of the presence of the semantic interference effect.

In Experiments 1 and 2 reported in Piai et al. (2015) we found that at the early SOA there was not enough evidence to support either the null or the alternate hypothesis. In comparison with the results from Experiment 2 reported in this paper, only a small amount of the data fell within the ROPE (16\% and 17\%), and a smaller proportion fell below zero. These results suggest that the data from Piai et al. (2015) are not strong enough to suggest that the absence of semantic interference supports the null hypothesis. ${ }^{3}$

\footnotetext{
${ }^{3}$ We also re-analysed Piai et al. (2015)'s data using linear mixed effects models, rather than repeated measures ANOVAs. In this way we were able to
} 
Table 7

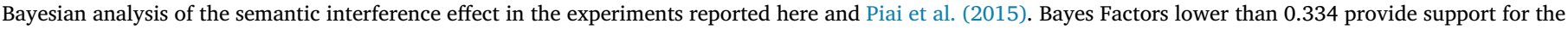

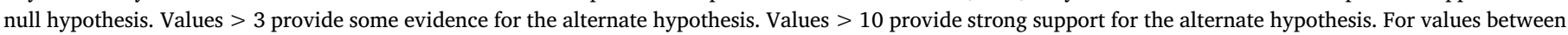

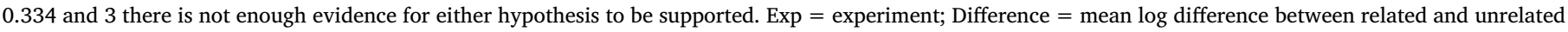

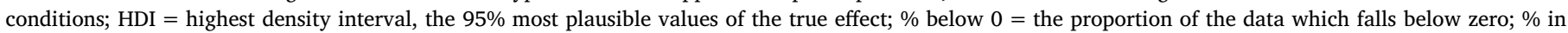

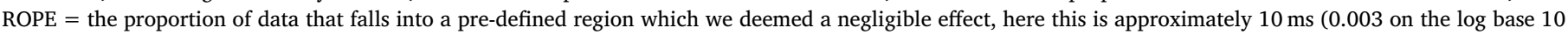
scale); $\mathrm{BF}=$ Bayes Factor.

\begin{tabular}{|c|c|c|c|c|c|}
\hline Experiment & Difference & 95\% HDI & $\%$ below 0 & $\%$ in ROPE & BF \\
\hline Exp 1: S1 syllable, $0 \mathrm{~ms}$ & 0.013 & $0.007,0.018$ & 0 & 0 & 284.47 \\
\hline Exp 1: $\mathrm{S} 1$ tone, $0 \mathrm{~ms}$ & 0.009 & $0.003,0.015$ & 0.2 & 3 & 7.67 \\
\hline Exp 1: S1 syllable, $1000 \mathrm{~ms}$ & 0.01 & $0.004,0.016$ & 0 & 1 & 39.3 \\
\hline $\operatorname{Exp} 1: \mathrm{S} 1$ tone, $1000 \mathrm{~ms}$ & 0.012 & $0.006,0.019$ & 0 & 0 & 60.36 \\
\hline Exp 2: S1 syllable, $0 \mathrm{~ms}$ & 0.013 & $0.003,0.023$ & 0.6 & 2 & 2.2 \\
\hline Exp 2: $\mathrm{S} 1$ tone, $0 \mathrm{~ms}$ & 0.0007 & $-0.009,0.009$ & 49.9 & 51 & 0.1 \\
\hline Exp 2: S1 syllable, $1000 \mathrm{~ms}$ & 0.015 & $0.006,0.023$ & 0.1 & 0 & 30.73 \\
\hline Exp 2: $\mathrm{S} 1$ tone, $1000 \mathrm{~ms}$ & 0.002 & $-0.007,0.011$ & 29.4 & 43 & 0.11 \\
\hline Exp 3: S1 syllable, $0 \mathrm{~ms}$ & 0.01 & $0.0007,0.019$ & 1.9 & 7 & 0.75 \\
\hline Exp 3: $\mathrm{S} 1$ tone, $0 \mathrm{~ms}$ & 0.009 & $0.0005,0.017$ & 1.9 & 8 & 0.94 \\
\hline Exp 3: S1 syllable, $1000 \mathrm{~ms}$ & 0.013 & $0.004,0.023$ & 0.3 & 1 & 4.09 \\
\hline Exp 3: $\mathrm{S} 1$ tone, $1000 \mathrm{~ms}$ & 0.008 & $0.0002,0.016$ & 2.3 & 9 & 0.7 \\
\hline Piai et al. (2015): $\operatorname{Exp} 1,0 \mathrm{~ms}$ & 0.007 & $-0.002,0.017$ & 6.2 & 16 & 0.51 \\
\hline Piai et al. (2015): $\operatorname{Exp} 1,1000 \mathrm{~ms}$ & 0.023 & $0.013,0.034$ & 0 & 0 & 204.56 \\
\hline Piai et al. (2015): $\operatorname{Exp} 2,0 \mathrm{~ms}$ & 0.007 & $-0.003,0.018$ & 7.4 & 17 & 0.37 \\
\hline Piai et al. (2015): $\operatorname{Exp} 2,350 \mathrm{~ms}$ & 0.019 & $0.011,0.027$ & 0 & 0 & 87.02 \\
\hline
\end{tabular}

In sum, Experiment 1 of the present study, which used the PRP paradigm, provided solid evidence for the presence of semantic interference at both SOAs and regardless of whether the first stimulus was a tone or a syllable. In line with many earlier studies, this indicates that the participants first selected the response to the auditory stimulus and then selected the name of the picture. The remaining experiments, using the TC paradigm mostly provided solid evidence for the presence of semantic interference at the $1000 \mathrm{~ms}$ SOA. The only exception was the non-linguistic condition of our Experiment 2, where we found solid evidence for the absence of such an effect. As noted above, it is not clear why the effect was absent in this condition. More importantly, the results obtained for the $0 \mathrm{~ms}$ SOA are inconclusive. None of the TC studies examined here provided convincing evidence for the presence or for the absence of a semantic interference effect at this SOA (except the nonlinguistic condition in Experiment 2, but again it is unclear why this was the case). Thus, on the basis of these data, no general claims concerning the way participants schedule the processing of the first and second stimuli can be made.

\section{General discussion}

The aim of this study was to examine how participants coordinated lexical selection for a picture name with the processing of a concurrent tone or syllable. In Experiment 1, the psychological refractory period (PRP) paradigm was used to study whether lexical selection could occur in parallel with the selection of the response to the auditory stimulus. Experiments 2 and 3 used the task choice (TC) paradigm to determine whether lexical selection could occur concurrently with the processing of an auditory stimulus which determined the type of response, either picture naming or distractor reading. Below we first focus on the results of Experiment 1, and then turn to those of Experiments 2 and 3 and the Bayesian analysis.

\section{(footnote continued)}

concurrently control for participant and item random effects. The mixed model analysis found the same pattern of results for Experiment 1 as reported in Piai et al. (2015), but did not find the same results for Experiment 2. Specifically, we found a main effect of relatedness $(t=2.42)$, and no relatedness by SOA interaction $(t=1.42)$. This indicates that when accounting for participant and item variation, there is evidence for a semantic interference effect at SOA $0 \mathrm{~ms}$ in Experiment 2.
In Experiment 1, semantic interference effects were observed at both SOAs. We interpret this pattern within the framework of dual task theories (Meyer \& Kieras, 1997; Navon \& Miller, 1987; Pashler, 1994; Pashler \& Sutherland, 1998; Tombu \& Jolicœur, 2003; see Fig. 1). Preselection processes, i.e. visual and early conceptual processes, occurred in parallel for the auditory stimulus and the picture. However, the selection of the response to the tone or syllable and the selection of the picture name occurred in sequence. The semantic interference effect arose because lexical selection took more time in the related than in the unrelated condition. If lexical selection had occurred in parallel with the processing and the response selection for the auditory stimulus, the interference effect would have been absorbed into cognitive slack created by these concurrent processes. In other words, the presence of the semantic interference effect at both SOAs shows that lexical selection followed the selection of the response to the tone or syllable. Our results replicate those of previous PRP studies using similar stimuli and designs (e.g., Ayora et al., 2011; Piai et al., 2011, 2014; Schnur \& Martin, 2012). However, in the earlier studies the first stimulus was a tone. Here, we have shown that the same pattern of results was obtained when the first stimulus was a syllable. This is important because PRP research can potentially contribute to our understanding of the way interlocutors coordinate listening and speech planning in conversation. In order to use the paradigm in this context, we first needed to establish whether basic findings obtained with the PRP paradigm can be replicated when the two tasks are linguistic. Our Experiment 1 shows that this is the case.

Additionally we found, not only in Experiment 1 but in all experiments, that at the $0 \mathrm{~ms}$ SOA the picture naming latencies were longer when the first stimulus was a syllable than when it was a tone. In the statistical analyses of the picture naming latencies, the RTs to the tones or syllables were controlled for. Therefore, it was not the case that lexical selection was simply initiated and completed later because the preceding processes of response selection occurred later for syllables than for tones. At the $1000 \mathrm{~ms}$ SOA, picture naming latencies after tones and syllables did not differ from one another. This pattern indicates that concurrently presented syllables interfered more with picture naming than tones. In Experiment 2 this was also true for distractor word reading. Most likely additional interference arose in the linguistic condition because the syllables, but not the tones, activated linguistic representations that were relevant to the naming and reading task. The nature of the relevant representations and the precise origin of this cross-talk effect need to be determined in future work. Given that the 
auditory stimuli were syllables but not words of the participants' native language, it is most likely that the implicated representations were phonological (rather than lexical) and that the effect arose late, during the generation of the phonological forms of the picture names. Fargier and Laganaro (2016) also conducted a dual-task study involving tone or syllable identification as task 1 and picture naming as task 2, and likewise reported longer picture naming latencies in the syllable than the tone condition. The EEG results obtained in this study pointed towards a late, phonological origin of this effect. However, in Fargier and Laganaro's study the syllables corresponded to words of the participants' language; hence additional interference may have occurred at the lexical level. In the present study, the syllables were not words, but they may nevertheless have activated word meanings through spreading activation (Gaskell \& Marslen-Wilson, 1997, 2002). The importance of phonological and lexical interference in dual-tasking with two linguistic tasks can now be explored in future work using the same paradigm. Such research would importantly contribute to attaining the goal of understanding the coordination of speaking and listening in everyday conversations.

The PRP experiment (Experiment 1) yielded robust evidence for the presence of semantic interference effects at both SOAs, in the presence of tones as well as syllables. Though there are some exceptions (Dell'Acqua et al., 2007), this pattern of results is in line with the findings reported in earlier studies (e.g., Piai et al., 2014; Schnur \& Martin, 2012). The majority of the evidence indicates that response selection for the auditory stimulus precedes the selection of a picture name. The stable pattern of results seen for PRP experiments contrasts sharply with the variable pattern seen in TC experiments compared above (Table 7, Experiments 2 and 3, Piai et al. (2015)'s Experiments 1 and 2). The most consistent finding across the TC experiments was the semantic interference effect at the $1000 \mathrm{~ms} \mathrm{SOA}$, though even this effect was not seen in the non-linguistic condition of our Experiment 2. By contrast the effects seen at the $0 \mathrm{~ms}$ SOA were highly variable and do not constitute convincing evidence for the presence or for the absence of semantic interference. Consequently, no firm conclusions can be drawn about the research question motivating the use of this paradigm, namely whether lexical selection co-occurred with the early processing of the first stimulus.

Other TC experiments assessing the presence of semantic interference have likewise yielded inconsistent findings. Janssen et al. (2008) presented target pictures with distractor words written in blue or red ink. Depending on the ink colour, participants read the distractor aloud or named the picture. SOAs of $0 \mathrm{~ms}$ and $1000 \mathrm{~ms}$ were used, and semantic interference effects were found at both SOAs. In contrast, Mädebach et al. (2011) used the same method and found no semantic interference effect at the $0 \mathrm{~ms}$ SOA. Several studies (O'Malley \& Besner, 2011; Paulitzki et al., 2009) have used the TC paradigm in conjunction with word and non-word reading tasks in order to study whether lexical and/or prelexical processes can co-occur with the processing of the task choice cue, and have likewise generated somewhat inconsistent findings.

It is always difficult to establish why some paradigms appear to yield more stable results than others. With respect to the PRP and TC paradigms, the comparison made in Table 7 is instructive because all studies recruited participants from the same academic community, used very similar materials and equipment, and were closely matched in number of observations. The main difference between the paradigms is obviously the task. We propose that the PRP paradigm is likely to yield more stable results (i.e. conclusive evidence from individual studies and consistency across studies) because the participants are explicitly instructed about the order of processing the auditory and visual stimuli and, perhaps more importantly, their speed of processing the auditory task is monitored.

To elaborate, on each trial of our PRP experiment, participants were presented with three stimuli: an auditory stimulus, a picture, and a distractor word. They were explicitly instructed to respond to the auditory stimulus before naming the picture and to ignore the distractor. These instructions held for all trials and the participants were aware that their performance on both tasks, identification of the auditory stimulus and picture naming, was monitored. Consequently, they should have been highly and consistently motivated to first prioritize processing of the auditory stimulus and then turn to the picture, ignoring the distractor word as much as possible.

In the TC paradigm, the same stimuli were presented, but no overt response to the auditory stimulus was required. However, this stimulus was response-relevant, as it determined how to respond to the visual stimulus, by reading the distractor or naming the picture. Thus, the picture name and distractor word were equally as important on each trial. Piai et al. (2011) discussed how task decision and speech planning processes could be scheduled relative to each other. As explained above, they proposed that speakers initially prepared responses to the picture and to the distractor word in parallel, but then suspended these processes to make the task choice. Based on earlier evidence (Reynolds \& Besner, 2006; Roelofs, 2008) they argued that a good suspension point would be prior to word form encoding. This implies that lexical selection for picture names occurred prior to suspension and semantic interference was absorbed into the cognitive slack created by the task choice.

In line with Piai and colleagues' proposal, there are two ways to schedule task processes in the TC paradigm. One way is to make the task choice as early as possible and then proceed with the encoding of either the word or the picture. This strategy should minimize the amount of linguistic encoding carried out in parallel for both stimuli. If such a strategy is adopted, lexical selection for the target picture may not have occurred before task suspension, and consequently a semantic interference effect should be measured. In contrast, if the task choice is made slowly (creating cognitive slack), lexical selection should be completed and no semantic interference effect should be measured. In other words, whether or not a semantic interference effect is observed will depend on how strongly and consistently participants strive to make an early task decision in each trial. The same line of reasoning holds for the PRP paradigm: Task 1 only creates sufficient cognitive slack to absorb differences in the speed of concurrent processes pertaining to task 2 when it takes long enough to complete. In the PRP paradigm, participants are clearly instructed on how to prioritize the tasks and performance on both tasks is monitored. In the TC paradigm participants have to develop a processing strategy themselves and they may vary across trials on how early they make the task decision. We suggest that the higher degree of uniformity of the processing strategies enforced in the PRP paradigm may importantly contribute to the higher consistency of the response strategies within and across participants and, ultimately, of the results across studies.

For psycholinguistic research, the TC paradigm is appealing precisely because it does not force participants to respond overtly to the stimuli they hear. This renders the paradigm more similar to dualtasking in conversation, where interlocutors listen to others and simultaneously prepare their utterances (Barthel et al., 2016; Bögels et al., 2015; Levinson \& Torreira, 2015). However, the dependency of the results on the participants' variable response strategies is problematic. In future research one might aim to develop versions of the TC paradigm addressing this issue. For instance, one could explicitly instruct participants to prioritize processing one of the stimuli, or one could force them to attend early to the pictorial stimulus by presenting it for a very brief period of time. In addition, one might use neurobiological indicators of the allocation of attention to the stimuli (for review see Luck, Woodman, \& Vogel, 2000) or the onset of response selection (e.g., Lien et al., 2007).

In conclusion, this study had two goals: (1) to explore the usefulness of dual-task paradigms for research on the coordination of speaking and listening, and (2) to examine how lexical selection for picture naming was combined with the processing of syllables and tones. With respect to the first goal, we found that both paradigms used here were equally 
as useful for syllable as for tone identification as task 1. Thus, both paradigms can be used in future psycholinguistic research, though the task choice paradigm should be adapted to increase the uniformity of the participants' response strategies or/and to trace these strategies. Concerning the second goal, we did not observe that syllable identification encouraged participants to alter their processing strategy dramatically compared to tone identification. We did however find that syllables interfered more with picture naming than tones. This might not seem too surprising, but is remarkable since only two syllables, not corresponding to words, were used and presented over many trials. One might have thought that under these circumstances the linguistic or non-linguistic nature of the auditory stimuli would not matter much. We found, however, that it did matter, and that the syllables consistently interfered more with picture naming. Future dual-task work can further explore the origin of this cross-talk effect and determine how properties of concurrent speech comprehension affect speech planning.

\section{Acknowledgements}

We are grateful to Vitoria Piai for sharing her data and materials, and for being available for many discussions, to Phillip Alday for statistical advice, and to two anonymous reviewers for their valuable comments on the manuscript.

\section{Funding}

This research was supported by the Max Planck Society for the Advancement of Science, Munich, Germany.

\section{Conflict of interest statement}

The authors have no conflicts of interest to declare.

\section{Appendix A. Supplementary data}

Supplementary data to this article can be found online at https:// doi.org/10.1016/j.actpsy.2018.09.006.

\section{References}

Ayora, P., Peressotti, F., Alario, F.-X., Mulatti, C., Pluchino, P., Job, R., \& Dell'Acqua, R. (2011). What phonological facilitation tells about semantic interference: A dual-task study. Frontiers in Psychology, 2(57), 1-10.

Barr, D. J., Levy, R., Scheepers, C., \& Tily, H. J. (2013). Random effects structure for confirmatory hypothesis testing: Keep it maximal. Journal of Memory and Language, 68(3), 255-278.

Barthel, M., Sauppe, S., Levinson, S. C., \& Meyer, A. S. (2016). The timing of utterance planning in task-oriented dialogue: Evidence from a novel list-completion paradigm. Frontiers in Psychology, 7, 1858.

Bates, D., Maechler, M., Bolker, B., Walker, S., et al. (2014). lme4: Linear mixed-effects models using eigen and $s 4$. R package version, 1(7).

Besner, D., \& Care, S. (2003). A paradigm for exploring what the mind does while deciding what it should do. Canadian Journal of Experimental Psychology/Revue canadienne de psychologie expérimentale, 57(4), 311-320.

Besner, D., \& Risko, E. F. (2005). Stimulus-response compatible orienting and the effect of an action not taken: Perception delayed is automaticity denied. Psychonomic Bulletin \& Review, 12(2), 271-275.

Boersma, P., et al. (2002). Praat, a system for doing phonetics by computer. Glot International, 5(9/10), 341-345.

Bögels, S., Magyari, L., \& Levinson, S. C. (2015). Neural signatures of response planning occur midway through an incoming question in conversation. Scientific Reports, 5, 12881.

Brown, T. L., Joneleit, K., Robinson, C. S., \& Brown, C. R. (2002). Automaticity in reading and the stroop task: Testing the limits of involuntary word processing. The American Journal of Psychology, 115(4), 515-543.

Cleland, A. A., Tamminen, J., Quinlan, P. T., \& Gaskell, M. G. (2012). Spoken word processing creates a lexical bottleneck. Language \& Cognitive Processes, 27(4), 572-593.

Cook, A. E., \& Meyer, A. S. (2008). Capacity demands of phoneme selection in word production: New evidence from dual-task experiments. Journal of Experimental Psychology: Learning, Memory, and Cognition, 34(4), 886-899.

Damian, M. F., \& Martin, R. C. (1999). Semantic and phonological codes interact in single word production. Journal of Experimental Psychology: Learning, Memory, and Cognition, 25(2), 345-361.

Dell'Acqua, R., Job, R., Peressotti, F., \& Pascali, A. (2007). The picture-word interference effect is not a stroop effect. Psychonomic Bulletin \& Review, 14(4), 717-722.

Fairs, A., Bögels, S., \& Meyer, A. S. (2018). Interference in dual-tasking simple linguistic tasks may be due to linguistic interference or acoustic complexity. (in preparation).

Fargier, R., \& Laganaro, M. (2016). Neurophysiological modulations of non-verbal and verbal dual-tasks interference during word planning. PLoS One, 11(12), e0168358.

Ferreira, V. S., \& Pashler, H. (2002). Central bottleneck influences on the processing stages of word production. Journal of Experimental Psychology: Learning, Memory, and Cognition, 28(6), 1187-1199.

Gaskell, M. G., \& Marslen-Wilson, W. D. (1997). Integrating form and meaning: A distributed model of speech perception. Language \& Cognitive Processes, 12(5-6), 613-656.

Gaskell, M. G., \& Marslen-Wilson, W. D. (2002). Representation and competition in the perception of spoken words. Cognitive Psychology, 45(2), 220-266.

Glaser, W. R., \& Düngelhoff, F.-J. (1984). The time course of picture-word interference. Journal of Experimental Psychology: Human Perception and Performance, 10(5), 640-654.

Hommel, B. (1998). Automatic stimulus-response translation in dual-task performance. Journal of Experimental Psychology: Human Perception and Performance, 24(5), 1368-1384.

Janssen, N., Schirm, W., Mahon, B. Z., \& Caramazza, A. (2008). Semantic interference in a delayed naming task: Evidence for the response exclusion hypothesis. Journal of Experimental Psychology: Learning, Memory, and Cognition, 34(1), 249-256.

Kass, R. E., \& Raftery, A. E. (1995). Bayes factors. Journal of the American Statistical Association, 90(430), 773-795.

Kruschke, J. K., Aguinis, H., \& Joo, H. (2012). The time has come: Bayesian methods for data analysis in the organizational sciences. Organizational Research Methods, 15(4), $722-752$.

Kruschke, J. K., \& Meredith, M.. Best: Bayesian estimation supersedes the t-test [computer software manual]. (2017). Retrieved from https://CRAN.R-project.org/package= BEST ( $R$ package version 0.5.0).

Lenth, R. V. (2016). Least-squares means: The R package lsmeans. Journal of Statistical Software, 69(1), 1-33. https://doi.org/10.18637/jss.v069.i01.

Levinson, S. C., \& Torreira, F. (2015). Timing in turn-taking and its implications for processing models of language. Frontiers in Psychology, 6.

Lien, M.-C., Ruthruff, E., Hsieh, S., \& Yu, Y.-T. (2007). Parallel central processing between tasks: Evidence from lateralized readiness potentials. Psychonomic Bulletin \& Review, 14(1), 133-141.

Logan, G. D., \& Schulkind, M. D. (2000). Parallel memory retrieval in dual-task situations: I. semantic memory. Journal of Experimental Psychology: Human Perception and Performance, 26(3), 1072-1090.

Luce, P. A., \& Pisoni, D. B. (1998). Recognizing spoken words: The neighborhood activation model. Ear and Hearing, 19(1), 1-36.

Luck, S. J., Woodman, G. F., \& Vogel, E. K. (2000). Event-related potential studies of attention. Trends in Cognitive Sciences, 4(11), 432-440.

Mädebach, A., Oppermann, F., Hantsch, A., Curda, C., \& Jescheniak, J. D. (2011). Is there semantic interference in delayed naming? Journal of Experimental Psychology: Learning, Memory, and Cognition, 37(2), 522-538.

Marslen-Wilson, W. D. (1987). Functional parallelism in spoken word-recognition. Cognition, 25(1-2), 71-102.

Meyer, D. E., \& Kieras, D. E. (1997). A computational theory of executive cognitive processes and multiple-task performance: Part I. basic mechanisms. Psychological Review, 104(1), 3.

Miozzo, M., Pulvermüller, F., \& Hauk, O. (2015). Early parallel activation of semantics and phonology in picture naming: Evidence from a multiple linear regression meg study. Cerebral Cortex, 25(10), 3343-3355.

Morey, R. D., \& Rouder, J. N.. Bayesfactor: Computation of bayes factors for common designs [computer software manual]. (2015). Retrieved from https://CRAN.R-project.org/ package $=$ BayesFactor $(\mathrm{R}$ package version 0.9.12-2).

Navon, D., \& Miller, J. (1987). Role of outcome conflict in dual-task interference. Journal of Experimental Psychology: Human Perception and Performance, 13(3), 435-448.

O'Malley, S., \& Besner, D. (2011). Lexical processing while deciding what task to perform: Reading aloud in the context of the task set paradigm. Consciousness and Cognition, 20(4), 1594-1603.

Pashler, H. (1984). Processing stages in overlapping tasks: Evidence for a central bottleneck. Journal of Experimental Psychology: Human Perception and Performance, 10(3), 358-377.

Pashler, H. (1994). Dual-task interference in simple tasks: Data and theory. Psychological Bulletin, 116(2), 220-244.

Pashler, H., \& Sutherland, S. (1998). The psychology of attention. Vol. 15. Cambridge, MA: MIT Press.

Paucke, M., Oppermann, F., Koch, I., \& Jescheniak, J. D. (2015). On the costs of parallel processing in dual-task performance: The case of lexical processing in word production. Journal of Experimental Psychology: Human Perception and Performance, 41(6), 1539-1552.

Paulitzki, J. R., Risko, E. F., O'Malley, S., Stolz, J. A., \& Besner, D. (2009). On the role of set when reading aloud: A dissociation between prelexical and lexical processing. Consciousness and Cognition, 18(1), 135-144.

Piai, V., Roelofs, A., \& Schriefers, H. (2011). Semantic interference in immediate and delayed naming and reading: Attention and task decisions. Journal of Memory and Language, 64(4), 404-423.

Piai, V., Roelofs, A., \& Schriefers, H. (2014). Locus of semantic interference in picture naming: Evidence from dual-task performance. Journal of Experimental Psychology: Learning, Memory, and Cognition, 40(1), 147. 
Piai, V., Roelofs, A., \& Schriefers, H. (2015). Task choice and semantic interference in picture naming. Acta Psychologica, 157, 13-22.

R Core Team (2014). R: A language and environment for statistical computing [computer software manual]. Vienna, Austria. Retrieved from http://www.R-project.org/.

Reynolds, M., \& Besner, D. (2006). Reading aloud is not automatic: Processing capacity is required to generate a phonological code from print. Journal of Experimental Psychology: Human Perception and Performance, 32(6), 1303-1323.

Risko, E. F., \& Besner, D. (2008). A role for set in the control of automatic spatial response activation. Experimental Psychology, 55(1), 38-46.

Roelofs, A. (1992). A spreading-activation theory of lemma retrieval in speaking. Cognition, 42(1-3), 107-142.

Roelofs, A. (2003). Goal-referenced selection of verbal action: Modeling attentional control in the stroop task. Psychological Review, 110(1), 88.

Roelofs, A. (2008). Attention, gaze shifting, and dual-task interference from phonological encoding in spoken word planning. Journal of Experimental Psychology: Human Perception and Performance, 34(6), 1580-1598.
Schnur, T. T., \& Martin, R. (2012). Semantic picture-word interference is a postperceptual effect. Psychonomic Bulletin \& Review, 19(2), 301-308.

Schriefers, H., Meyer, A. S., \& Levelt, W. J. (1990). Exploring the time course of lexical access in language production: Picture-word interference studies. Journal of Memory and Language, 29(1), 86-102.

Sjerps, M. J., \& Meyer, A. S. (2015). Variation in dual-task performance reveals late initiation of speech planning in turn-taking. Cognition, 136, 304-324.

Strijkers, K., Costa, A., \& Pulvermüller, F. (2017). The cortical dynamics of speaking: Lexical and phonological knowledge simultaneously recruit the frontal and temporal cortex within 200 ms. NeuroImage, 163, 206-219.

Tombu, M., \& Jolicœur, P. (2003). A central capacity sharing model of dual-task performance. Journal of Experimental Psychology: Human Perception and Performance, 29(1), 3.

van Casteren, M., \& Davis, M. H. (2006). Mix, a program for pseudorandomization. Behavior Research Methods, 38(4), 584-589. 